\title{
Stress-based performance evaluation of osseointegrated dental implants by finite-element simulation
}

\author{
Luigi Baggi ${ }^{\mathrm{a}}$, Ilaria Cappelloni ${ }^{\mathrm{b}}$, Franco Maceri ${ }^{\mathrm{c}}$, Giuseppe Vairo ${ }^{\mathrm{c}, *}$ \\ a School of Dentistry, University of Rome "Tor Vergata", 00133 Roma, Italy \\ ${ }^{\mathrm{b}}$ Department of Mechanical Engineering, University of Rome "Tor Vergata", 00133 Roma, Italy

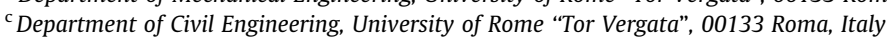

\section{A R T I C L E I N F O}

\section{Article history:}

Received 4 January 2008

Received in revised form 27 March 2008

Accepted 9 May 2008

Available online 10 July 2008

\section{Keywords:}

3D finite-element simulation

Dental biomechanics

Osseointegrated dental implants

Platform switching influence

Trabecular bone quality influence

\begin{abstract}
A B S T R A C T
In this paper biomechanical interaction between osseointegrated dental implants and bone is numerically investigated through 3D linearly elastic finite-element analyses, when static functional loads occur. Influence of some mechanical and geometrical parameters on bone stress distribution is highlighted and risk indicators relevant to critical overloading of bone are introduced. Insertions both in mandibular and maxillary molar segments are analyzed, taking into account different crestal bone loss configurations. Stress-based performances of five commercially-available dental implants are evaluated, demonstrating as the optimal choice of an endosseous implant is strongly affected by a number of shape parameters as well as by anatomy and mechanical properties of the site of placement. Moreover, effectiveness of some double-implant devices is addressed. The first one is relevant to a partially edentulous arch restoration, whereas other applications regard single-tooth restorations based on non-conventional endosteal mini-implants. Starting from computer tomography images and real devices, numerical models have been generated through a parametric algorithm based on a fully 3D approach. Furthermore, effectiveness and accuracy of finite-element simulations have been validated by means of a detailed convergence analysis.
\end{abstract}

(c) 2008 Elsevier B.V. All rights reserved.

\section{Introduction}

Use of osseointegrated dental implants allows restoration of completely or partially edentulous patients and success of this technique is closely linked to the direct connection between living bone and the surface of a load-bearing artificial structure, generally titanium-based. Endosteal implants can be usually employed to support single-tooth prostheses or fixed partial dentures. In this latter occurrence multiple-implant systems are generally used and a number of fixtures supports denture prosthesis by means of devices such as retaining bars, retaining balls, natural-like bridges.

As confirmed by several clinical studies [18,28,33,47,49,53,57], osseointegrated implants can fail mainly as a consequence of bone weakening or loss at peri-implant region. Moreover, clinical researches have highlighted that the number of implants in maxillary posterior region experiencing failure is generally higher than mandibular's one $[17,18,28,33,47]$.

Bone resorption process affects mainly the implant neck region and can be activated by surgical trauma or bacterial infection as well as by overloading at bone-implant interface $[6,8,27,49]$. Under functional forces, overloading of peri-implant

\footnotetext{
* Corresponding author. Tel.: +39 067259 7088; fax: +39 0672597005 .

E-mail address: vairo@ing.uniroma2.it (G. Vairo).
} 
bone can be induced by a shortcoming in load transfer mechanisms, mainly due to bad occlusion, incorrect implant use, wrong prosthesis and/or implant design, improper surgical placement. As a consequence, high stress concentrations may arise and, according to well-established hypotheses [8,15,27,39], the relating strain fields should stimulate biological bone resorption, jeopardising implant effectiveness. Nevertheless, clinical observations show as a significant reduction of bone loss at implant neck is possible when the connection diameter of the abutment is narrower than implant collars, that is when so called platform switching configurations are considered [19,32,37]. Reasons leading to bone preservation with platform switching have not yet been clarified but several hypotheses are relevant to the location of micro-gap between implant and abutment as well as to stress distributions at peri-implant regions [21,22]. Accordingly, it clearly appears as an accurate evaluation of stress distribution on bone allows to investigate about effectiveness and reliability of endosseous implants, revealing risks of implant failure $[42,43]$.

As a matter of fact, stress and strain fields around endosteal implants are strongly affected by a number of biomechanical factors, including type of loading, material properties of implant and prosthesis, implant geometry, surface structure, quality and quantity of surrounding bone, and nature of bone-implant interface [6]. As far as implant shape is concerned, main design parameters affecting load transfer mechanisms include implant diameter and length of bone-implant interface, as well as, in the case of threaded implants, thread pitch, shape and depth. In order to increase surfaces appointed for osseous integration, threaded implants are generally preferred to smooth cylindrical ones [40]. Depending on bone quality, surface treatments and thread geometry can significantly influence implant effectiveness, in terms of both initial implant stability and biomechanical nature of bone-implant interface after the healing process [14].

As a result of both market demands and scientific research, new concepts have been developed and many implant typologies are commercially available in different size, shape, materials and surfaces. As a number of researches in this field clearly shows, the wide range of implant applications as well as the continuous evolution of clinical indications and protocols in prosthetic dentistry make the stress analysis of the bone-implant structural system an actual, open and important issue. The complex geometry of the coupled biomechanical bone-implant system prevents the use of a closed-form approach for stress/strain evaluation and then numerical simulation methods are usually employed. In the last years, finite-element method [58] has been widely used in applied dentistry for analyzing both restorative techniques $[2,3,29,36]$ and implant applications [20,56], investigating influence of implant and prosthesis design [10,23,24,46], of magnitude and direction of loads [5,12,24,26], of bone mechanical properties [13,30], as well as modelling different clinical scenarios $[9,11,44,50,51]$. It is worth noting that influence of crestal bone loss configurations and of more detailed geometrical models for implant and bone have been usually disregarded in a number of recent studies $[5,12,23,24,26,30,37,50]$, where axisymmetric or two-dimensional simplified approaches are involved. More realistic results should be obtained through a more detailed 3D modelling for implant devices and site of placement (this latter clearly patient-dependent).

In this paper a number of endosteal dental implant applications are analyzed by means of statical linearly elastic finite-element simulations, based on a fully three-dimensional approach. In agreement with the clinical evidence after an healing period [7,52] and with the aim to understand some biomechanical aspects and advantages relevant to platform switching configurations, different compact bone geometries around implant neck are modelled, depending on crestal bone loss induced by implant shape. Moreover, in order to perform stress-based comparative evaluation of implant performance, risk indicators relevant to critical overloading of bone are introduced.

Firstly, use of five commercially-available osseointegrated dental implants is numerically investigated, highlighting biomechanical interaction between implant and bone as well as influence of some mechanical and geometrical parameters on load transfer mechanisms and on bone stress distributions. In order to investigate how the site of placement affects implant performance, insertions both in mandibular and maxillary molar segments are considered and stress distributions are numerically evaluated at peri-implant region on both compact and cancellous bone, giving risk measures of bone physiological failure. Moreover, in the case of mandibular insertions and considering available experimental results [48], influence of trabecular bone quality on implant stress-based performance is analyzed.

Afterwards, effectiveness of some multiple-implant systems is addressed. The first one is relevant to the case of a partially edentulous arch restoration and it is based on a double-implant device involving a gold-alloy retaining bar, supporting the prosthetic denture, fixed with two endosteal implants [4]. Two other applications regard the case of single-tooth restorations based on a non-conventional device, consisting in a titanium mini-bar supported by two endosteal mini-fixtures. These latter can be suitably angled (two cases are numerically investigated) in order to reproduce natural roots orientation in multi-root teeth.

It is worth observing that mini-implants are generally used in clinical orthodontic or skeletal applications, when temporary but absolute anchorages should be ensured [45] without complete osseous integration. Therefore, use of osseointegrated mini-implants for prosthetic dentistry applications can be considered as a novel clinical concept. In detail, using two small fixtures instead of a greater one (in terms of both insertion length and diameter) should be advantageous when geometrical configuration of the site of placement and/or bone quantity and quality (particularly in sinus zone) do not allow to employ traditional implants ensuring long-term success and/or an effective healing process. Some analyses of dental implants with reduced dimensions are available in specialized literature [1,25], but geometrical parameters of these smaller implants do not significantly differ from the traditional's ones, resulting not in agreement with typical mini-fixture dimensions (thread diameter ranging from $1.2 \mathrm{~mm}$ up to $2.5 \mathrm{~mm}$; insertion length from $4.0 \mathrm{~mm}$ to $12 \mathrm{~mm}[16,31,38])$. 


\section{Material and methods}

\subsection{Numerical models}

In this paper the use of five commercial threaded dental implants is numerically investigated (Fig. 1a): two standard ITI implants (Institute Straumann AG, Waldenburg, Switzerland), two Branemark implant systems (Nobel Biocare AB, Goteborg, Sweden), an Ankylos implant device (Dentsply Friadent, Mannheim, Germany).

As sketched in Fig. 1a, ITI devices and the first Branemark implant are modelled by single-body structures; the second Branemark implant is connected to the abutment by an internal screw; Ankylos system has a threaded abutment directly inserted into the fixture. Moreover, thread is trapezoidal for the Ankylos implant and triangular for all the other devices.

With reference to notation introduced in Fig. 1a and as summarized in Table 1, implant diameters vary from 3.3 mm to $4.5 \mathrm{~mm}$, whereas length of implant-bone interface is assumed between $7.5 \mathrm{~mm}$ and $12 \mathrm{~mm}$. Furthermore, all the analyzed implants are practically comparable in thread pitch and depth.

Starting from the model of the Ankylos device, solid models relevant to multi-implant applications are also carried out (Fig. 1b). In detail, a double-implant system (DIRB) able to support three molar prosthetic crowns is modelled considering a gold retaining bar, with a length of about $22 \mathrm{~mm}$, perfectly fixed with two parallel commercial Ankylos implants, whose inter-axis is about $18 \mathrm{~mm}$. Moreover, non-conventional single-tooth implant models are also built up (MI). In this case two not-commercially-available mini-fixtures, characterized by Ankylos geometry and whose main geometrical parameters are summarized in Table 1, are connected by a titanium-based mini-bar, whose length is about 8 mm. Two different models are considered. In the first one, Ankylos-type mini-implants are assumed to be parallel $\left(\mathrm{MI}_{0}\right)$, whereas in the second case they are symmetrically angled at $25^{\circ}\left(\mathrm{MI}_{25}\right)$ with respect to the vertical axis (i.e., orthogonal-to-bar axis). In both cases inter-axis

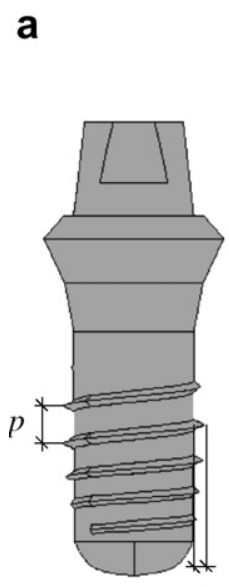

ITI 1

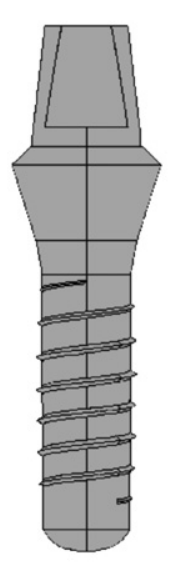

ITI 2

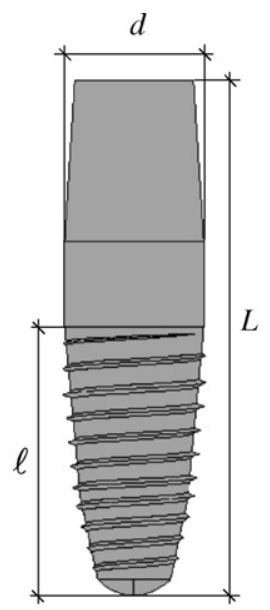

Branemark 1

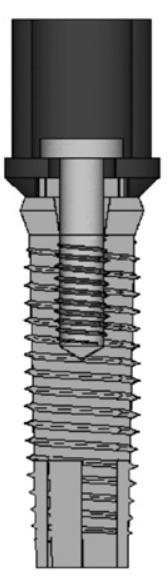

Branemark 2

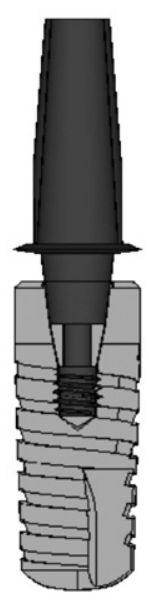

Ankylos
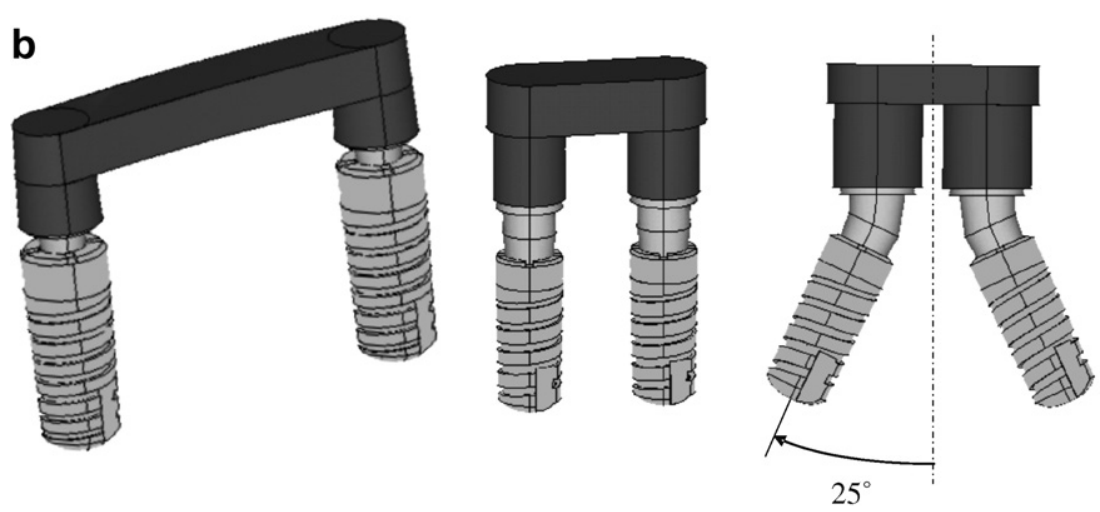

DIR B

MI 25

Fig. 1. Three-dimensional solid models of five commercial endosteal dental implants (a) and multi-implant devices based on Ankylos-type fixtures (b). DIRB: double-implant system with a gold retaining bar; MI: non-conventional mini-implant devices. 
Table 1

Main geometrical parameters (in $\mathrm{mm}$ ) for implants analyzed in this study

\begin{tabular}{lllll}
\hline Implant & $L$ & $\ell$ & $d$ & $p$ \\
\hline ITI 1 & 16 & 7.5 & 4.1 & 1.15 \\
ITI 2 & 17 & 9.0 & 3.3 & 0.98 \\
Branemark 1 & 16 & 9.0 & 4.5 & 0.24 \\
Branemark 2 & 14 & 12.0 & 3.75 & 0.20 \\
Ankylos & 11 & 11.0 & 4.5 & 0.21 \\
Mini-Ankylos & 6 & 6.0 & 2.5 & 0.27 \\
\end{tabular}

Notation refers to Fig. 1a: $L$ is the fixture total length; $\ell$ represents the assumed bone-implant interfacial length; $d$ indicates the implant maximum diameter; $p$ is the average thread pitch and $t$ is the average thread depth.

between abutments is equal to about $6 \mathrm{~mm}$ and abutment-fixture connection diameters are assumed to be smaller than implant collar ones, in agreement with platform switching concept.

In order to simulate a coupled bone-implant system, maxillary and mandibular bone segments relevant to molar regions are also modelled, neglecting the presence of gingival soft tissues. Bone segments are assumed to be composed by two volumes (Fig. 2): an outer shell with an average thickness of about $2 \mathrm{~mm}$ representing the cortical bone layer, and an inner volume representing the cancellous bone tissue, perfectly connected with the cortical's one. Length of bone segments along mesio-distal direction ( $y$ axis in Fig. 2) is about $40 \mathrm{~mm}$ for simulations of single-tooth implants and $60 \mathrm{~mm}$ for the DIRB device, whereas their average height is about $16 \mathrm{~mm}$ for the maxillary segment and $24 \mathrm{~mm}$ for the mandibular one. Implant systems are assumed to be approximatively placed at the mid-span of bone segments. Moreover, in order to reproduce in a realistic way the physiological bone structure after an healing period, different compact bone geometries around implant neck are modelled. In detail, depending on implant shape and in agreement with well-established clinical evidences [7,52] (Fig. 3a), two different compact bone geometries around implant neck are considered. As showed in Fig. 3b, a "flaring" shape is modelled for ITI and Branemark implants taking into account a mean crestal bone loss of about $45 \%$ in thickness, whereas for Ankylos device (both commercial and mini-implant type) platform switching configuration suggests to model a reduced crestal bone loss (assumed to be of about 25\% in thickness), with a cortical bone layer (of about $0.3 \mathrm{~mm}$ in thickness) following implant collar contour.

In order to generate all 3D solid models (bone segments and implants), starting from the numerical procedure developed by Vairo in [55] for modelling geometries of dental restorations, a parametric algorithm has been set up and implemented in a MatLab (The MathWorks, Inc., Natick, MA) homemade code, whose output is fully compatible with Ansys environment (Ansys, Inc., Canonsburg, PA). For implants, starting from high resolution pictures and real devices, several shape parameters (e.g., number and main dimensions of implant parts, thread geometry, etc.) are firstly identified. Successively, primitive cylindrical volumes relevant to each implant element (abutment, fixture, possible internal screw) are built up and, in order to reproduce the 3D primary topology of implant model, exceeding volumes with respect to real geometry are generated as union of simple volumes and removed from the primitive ones. As far as bone segments are concerned, starting from computer tomography (CT) images, a set of user-assigned points and physiological shape parameters for cancellous and compact bone is identified by SimPlant software (Materialise Dental, Inc., Leuven, Belgium). By means of a cubic interpolation algorithm on input data, significant primary profiles (lying in planes orthogonal to the mesio-distal direction, $y$ axis in Fig. 2) relevant to cortical and trabecular bone regions are reproduced. Afterwards, cubic-interpolated secondary profiles are created at different values of $y$ coordinate. Finally, dual cubic-interpolated profiles (orthogonal to $z$ axis) are added resulting in a wire-frame model from which bone segment volumes are generated (Fig. 2a).

The commercial tool Ansys 7.1 is used for merging the different parts comprising overall bone-implant models and for generating computational meshes. These employ ten-nodes tetrahedral elements based on a classical pure displacement formulation, with quadratic shape functions and three degrees of freedom per node [58]. As it will be explained in the following, for the same solid model several meshes have been generated, considering different mesh refinement levels close to periimplant regions and away from bone-implant interface.

\subsection{Material properties}

Under functional loads, dry-material models are employed for bone living tissues and therefore every fluid-solid interaction effect is neglected. All the involved materials are assumed with an isotropic linearly elastic behaviour and material volumes are considered as homogeneous. Implants, abutments and mini-bar of MI devices are assumed to be constituted by a titanium alloy, whereas retaining bar of DIRB system is modelled as made of a gold alloy. Referring to consolidated values available in literature, Table 2 summarizes the elastic properties used in this study. It is worth observing that, according to Lekholm and Zarb classification [34], material properties assumed for mandibular segment approximate a quality-II bone. On the other hand, as histologically confirmed and in agreement with Chun et al. [11], maxillary trabecular bone is assumed to be less dense than mandibular one and, accordingly, a smaller Young's modulus is employed [41].

In order to investigate about influence of trabecular bone quality on implant biomechanical response, different values of trabecular Young's modulus $\left(E_{\mathrm{t}}\right)$ are also considered for analyzing two commercial single-tooth implants (the first Branemark 
a

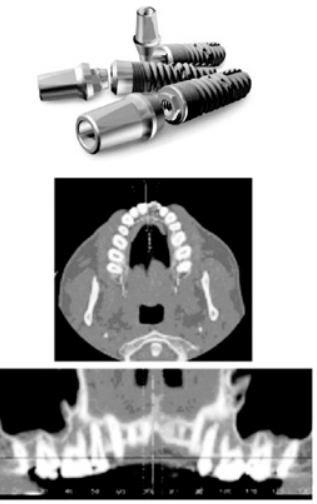

input data

b

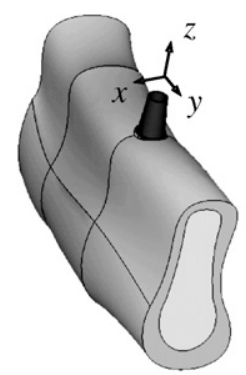

single-tooth commercial implants

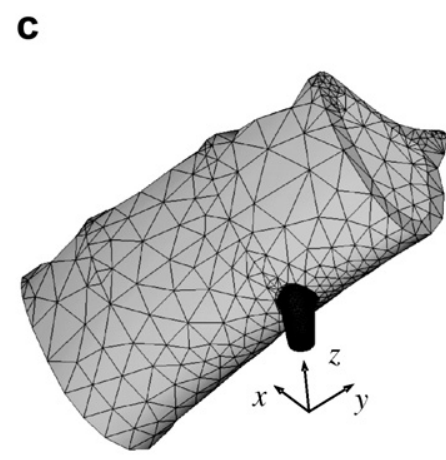

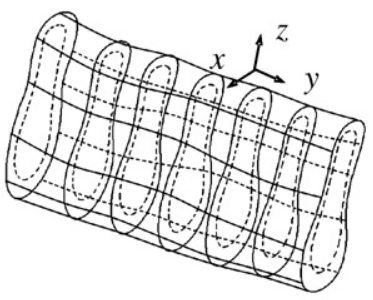

wire-frame models

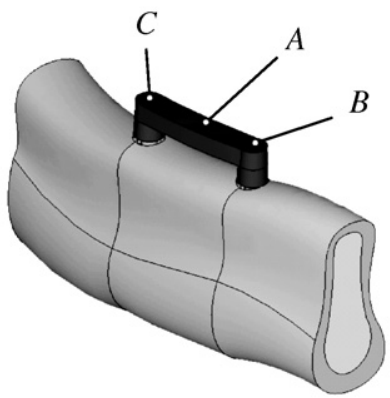

DIRB
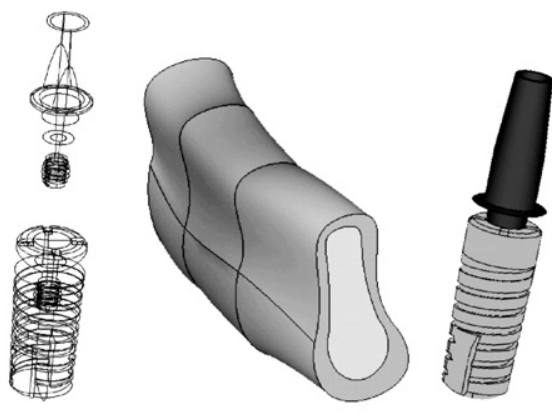

3D solid models

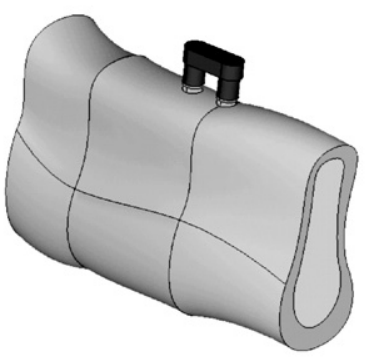

MI

maxillary molar segment

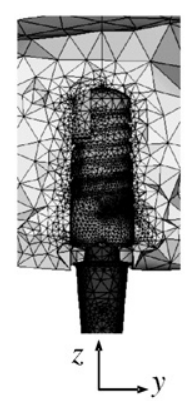

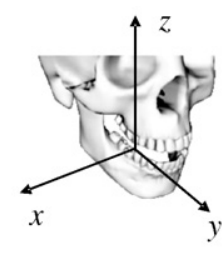
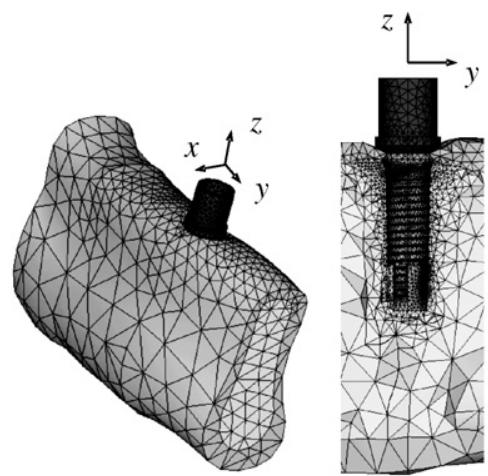

mandibular molar segment

Fig. 2. Three-dimensional numerical models: (a) generation process; (b) overall bone-implant solid models and (c) discretized details for both maxillary and mandibular bone segments. Notations.

and Ankylos) in mandibular applications. In detail, in addition to the case $E_{\mathrm{t}}=1.0 \mathrm{GPa}$ (Table 2) and in agreement with the experimental results proposed by Rho et al. [48], three values for $E_{\mathrm{t}}$ are also employed: $0.5 \mathrm{GPa}, 5.5 \mathrm{GPa}$ and $9.5 \mathrm{GPa}$, corresponding to a cancellous bone density ranging from about $0.5 \mathrm{~g} \mathrm{~cm}^{-3}$ to about $2.0 \mathrm{~g} \mathrm{~cm}^{-3}$.

\subsection{Loading and boundary conditions}

Finite-element simulations relevant to the five commercial single-tooth implants are carried out considering a functional load applied at the top of abutments without any eccentricity with respect to the vertical axis ( $z$ in Fig. 2 ), and angled at about $22^{\circ}$ to $z$. The lateral force component along bucco-lingual axis (opposed to the $x$-axis direction, see Fig. 2 ) is assumed equal to $100 \mathrm{~N}$ and the vertical intrusive one is $250 \mathrm{~N}$. This load is also considered in the case of DIRB and MI applications. As far as non-conventional mini-implant systems (MI) are concerned the force is applied at the mini-bar mid-span, whereas when DIRB system is experienced three different loading positions are considered on the upper-side bar centerline: at the 
a

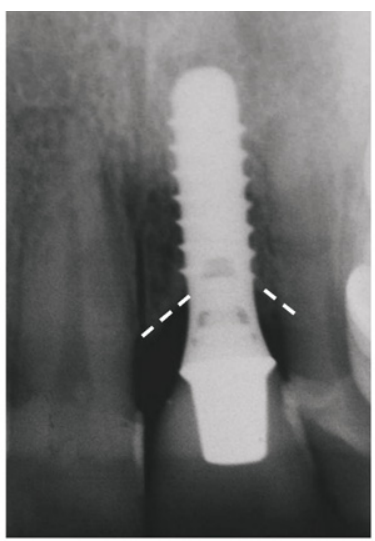

ITI 2

(Straumann)

b

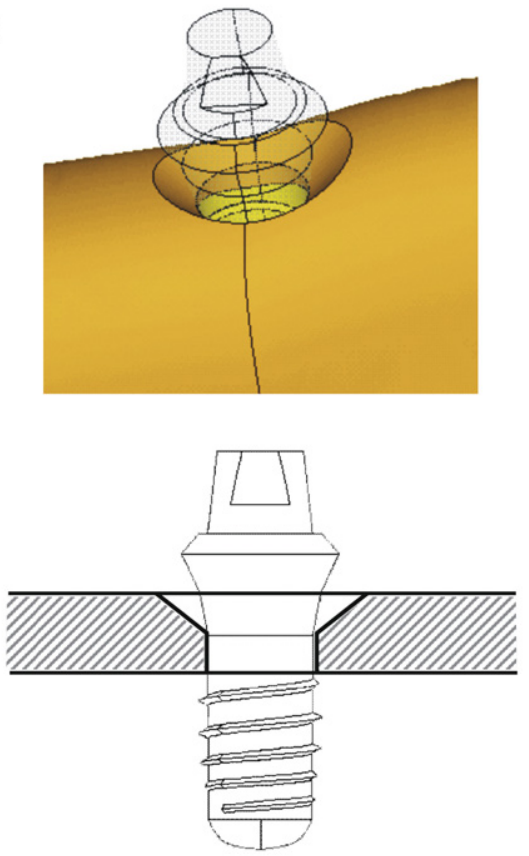

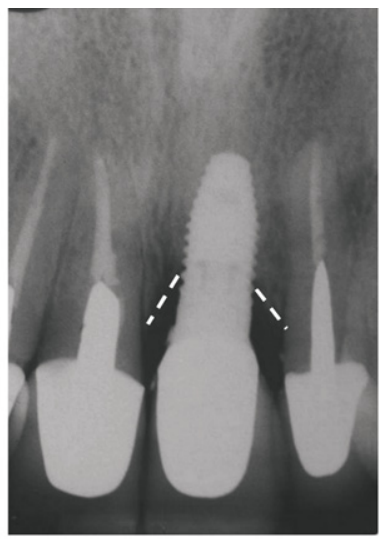

Branemark 1

(Nobel Biocare)

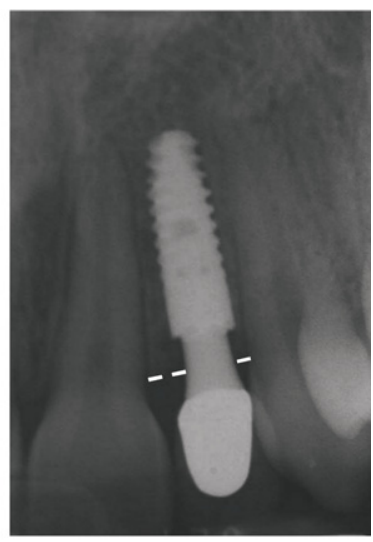

Ankylos

(Dentsply Friadent)

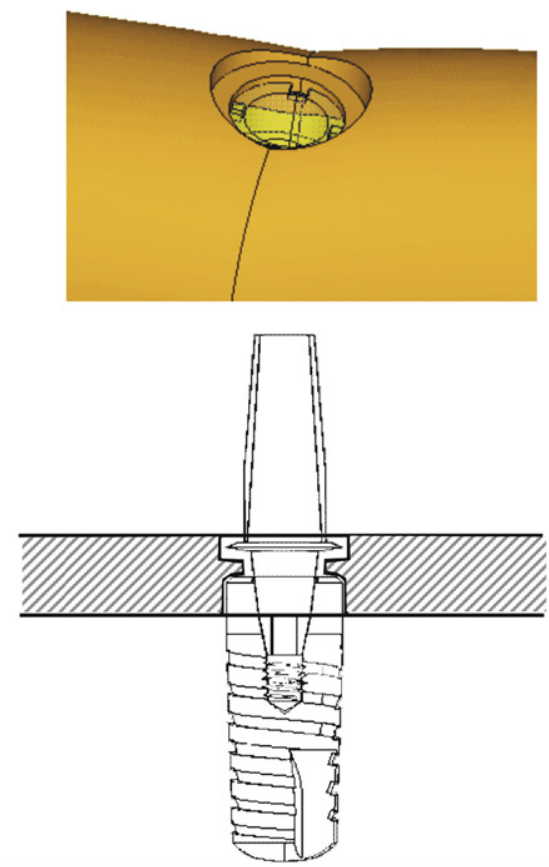

Fig. 3. Geometrical modelling of crestal bone loss induced by implant shape. Periapical radiographs showing crestal bone loss for Straumann, Nobel Biocare and Ankylos implants after a healing period of about 1 year (a); modelling of a significant "flaring" crestal bone loss (left) and of a reduced bone loss, due to platform switching configuration (right) (b).

mid-span (position A in Fig. 2) and at mesial and distal implant locations (positions B and C, respectively). In order to allow significant comparisons, abutments and bar-implant connections are adjusted in such a way that all loading application points are $7 \mathrm{~mm}$ far from the insertion bone surface.

Complete osseous integration between implants and natural tissues is assumed, enforcing as a displacement constraint the continuity of the displacement field at the implant-bone interface. Furthermore, displacement continuity is imposed between each component of implant devices. As a boundary condition for the coupled bone-implant system, all displacement degrees of freedom are prevented for any bone boundary node lying on end-sections parallel to the $x z$ plane. Since the free length of bone segments (i.e., the distance between end-surfaces of anatomical sites and implant location) is greater than the maximum implant dimension and in agreement with the theory of elasticity [54], these boundary conditions should not significantly affect stress results at peri-implant regions. 
Table 2

Elastic constants adopted for FE analyses

\begin{tabular}{|c|c|c|c|}
\hline Material & Region & $E(\mathrm{GPa})$ & $v$ \\
\hline Titanium alloy & Implants, abutments, mini-bar (MI) & $114.0^{\mathrm{a}, \mathrm{c}}$ & $0.34^{\mathrm{a}, \mathrm{c}}$ \\
\hline Gold alloy & Retaining bar (DIRB) & $105.0^{\mathrm{c}, \mathrm{e}}$ & $0.23^{\mathrm{c}, \mathrm{e}}$ \\
\hline \multirow[t]{2}{*}{ Cancellous bone } & Maxillary & $0.5^{\mathrm{b}},{ }^{\mathrm{d}}$ & $0.30^{\mathrm{b}, \mathrm{d}}$ \\
\hline & Mandibular & $1.0^{\mathrm{a}, \mathrm{d}}$ & $0.30^{\mathrm{b}, \mathrm{d}}$ \\
\hline Cortical bone & Maxillary and mandibular & $13.7^{\mathrm{a}, \mathrm{d}}$ & $0.30^{\mathrm{b}, \mathrm{d}}$ \\
\hline
\end{tabular}

$E$ is the Young's modulus and $v$ is Poisson's ratio.

a From Bozkaya et al. [5].

b From Chun et al. [11].

c From Lemon and Dietsh-Misch [35].

d Average values from Natali et al. [41].

e From Natali et al. [44].

\subsection{Stress measures and risk indicators}

For all the analyzed bone-implant systems stress distributions are numerically evaluated at peri-implant regions on both compact and cancellous bone, giving risk-measures of critical bone overloading. As it is customary in applied dentistry literature, Von Mises stress field $\sigma_{\mathrm{VM}}$ (always positive in sign) is used as a global stress measure for characterizing load transfer mechanisms of a given implant, whereas principal stresses $\left(\sigma_{i}\right.$, with $\left.i=1,2,3\right)$ are here employed as local risk measures of bone-implant interfacial physiological failure or of resorption process activation. In detail, if it is assumed as a physiological limit state that overload occurs when ultimate bone strength is reached, it follows that maximum principal compressive and tensile stress moduli on cortical bone should be less than 170-190 MPa and 100-130 MPa [39,41], respectively, whereas normal stress modulus on trabecular bone (both in compression and tension) should be less than about $5 \mathrm{MPa}$ [39].

With the aim to define quantitative stress measures useful for comparative evaluations and with reference to Fig. 4, let $\Omega_{\mathrm{t}}$ and $\Omega_{\mathrm{c}}$ be thin volumes with an average thickness of about $1.0 \mathrm{~mm}$ around a given implant and relevant to trabecular and cortical regions, respectively. Let $\Sigma_{\mathrm{t}}(z)$ be the two-dimensional region resulting from the intersection at a given value of $z$ between $\Omega_{\mathrm{t}}$ and a plane orthogonal to the implant axis. Moreover, let $\Sigma_{\mathrm{c}}(\theta)$ be the two-dimensional region resulting from the intersection between $\Omega_{\mathrm{c}}$ and a plane $\pi$ through the implant axis and identified by the angle $\theta$ with respect to the bucco-lingual axis $x$. In the case of multi-implant applications, volumes $\Omega_{\mathrm{c}}$ and $\Omega_{\mathrm{t}}$ are defined as the union of corresponding interfacial volumes relevant to each fixture.

Accordingly, the following Von Mises $\left(\sigma_{\mathrm{v}}\right)$ and principal $\left(\sigma_{\mathrm{C}}, \sigma_{\mathrm{T}}\right)$ stress measures can be introduced

$$
\begin{aligned}
\sigma_{\mathrm{v}}^{b}(\delta) & =\frac{1}{\mathscr{D}(\delta)} \int_{\mathscr{D}(\delta)} \sigma_{\mathrm{VM}}(x, y, z) \mathrm{d} a \\
\sigma_{\mathrm{T}}^{b}(\delta) & =\max _{\mathscr{D}(\delta), i=1,2,3}\left\{\sigma_{i}(x, y, z), 0\right\} \\
\sigma_{\mathrm{C}}^{b}(\delta) & =\min _{\mathscr{D}(\delta), i=1,2,3}\left\{\sigma_{i}(x, y, z), 0\right\}
\end{aligned}
$$

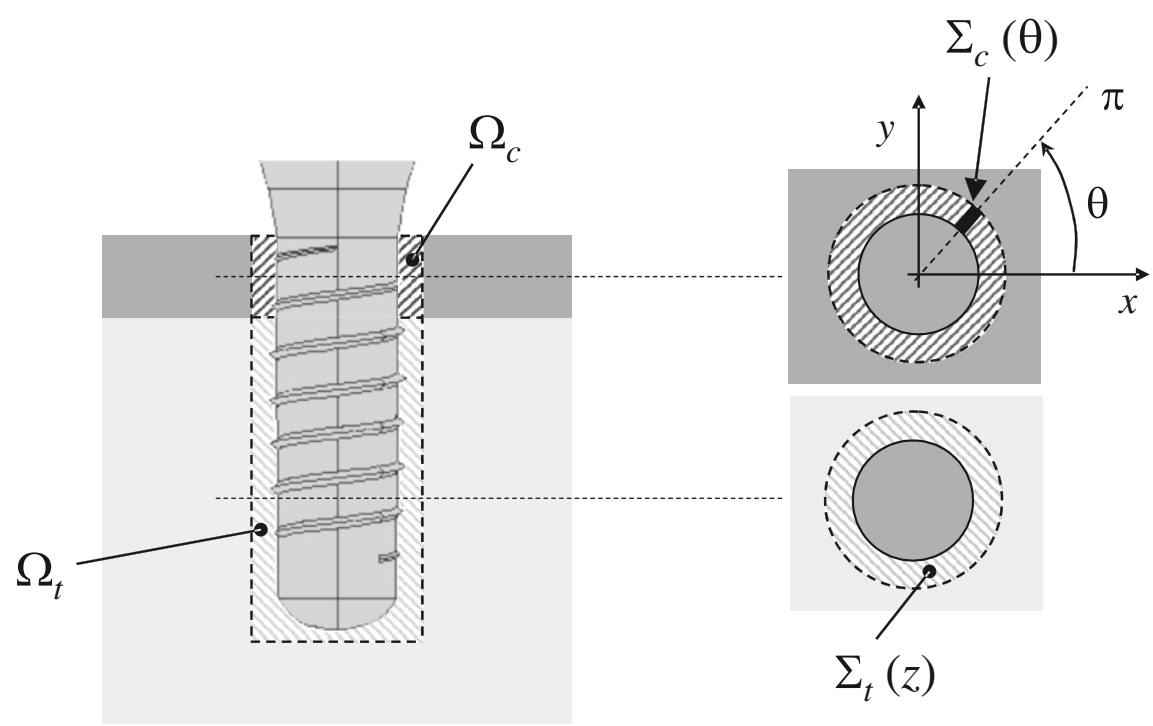

Fig. 4. Control regions employed for defining local stress measures at bone-implant interface. 
where the domain $\mathscr{D}(\delta)$ corresponds to $\Sigma_{\mathrm{t}}(z)$ for stress measures relevant to the trabecular peri-implant region $(\delta=z, b=t)$ and to $\Sigma_{\mathrm{c}}(\theta)$ for those defined at compact bone $(\delta=\theta, b=c)$.

It is worth observing that $\sigma_{\mathrm{v}}$ gives a measure of local mean stress distribution at implant-bone interface, whereas $\sigma_{\mathrm{T}}$ and $\sigma_{\mathrm{C}}$ represent overloading risk indicators at peri-implant regions with reference to tensile and compressive states, respectively.

The previously-introduced stress measures are numerically computed through a post-processing phase performed by means of a MatLab homemade procedure, taking as input by the solver code some primary geometrical and topological data (i.e., nodal coordinates and elements lying at bone-implant interfacial regions $\Omega_{\mathrm{t}}$ and $\Omega_{\mathrm{c}}$ ) as well as stress solutions at finiteelement integration points.

\section{Results}

\subsection{Validation}

In order to ensure accurate numerical results at peri-implant regions, mesh-size $h$ for coupled bone-implant models is set up on the basis of a preliminary convergence analysis. In detail, models' discretization is made at four different mesh refinement levels: $h_{\mathrm{o}}=3.0 \mathrm{~mm}$ and $h_{i}=0.8 \mathrm{~mm}(\mathrm{M} 1), h_{\mathrm{o}}=1.5 \mathrm{~mm}$ and $h_{i}=0.4 \mathrm{~mm}(\mathrm{M} 2), h_{\mathrm{o}}=0.8 \mathrm{~mm}$ and $h_{i}=0.2 \mathrm{~mm}(\mathrm{M} 3)$, $h_{\mathrm{o}}=0.6 \mathrm{~mm}$ and $h_{i}=0.1 \mathrm{~mm}(\mathrm{M} 4), h_{\mathrm{o}}$ and $h_{i}$ being mean mesh-sizes away from bone-implant interface and close to peri-implant regions, respectively. In order to furnish error estimates on a given computational domain $\Omega$, relative displacement and energy error norms can be respectively introduced [58]:

$$
E_{u}=\frac{\left\|e_{u}\right\|}{\|u\|}, \quad E_{\varepsilon}=\frac{\left\|e_{\varepsilon}\right\|}{\|\varepsilon\|}
$$

with

$$
\begin{aligned}
\left\|e_{u}\right\|^{2} & =\int_{\Omega}\left(\mathbf{u}_{h}-\hat{\mathbf{u}}\right) \cdot\left(\mathbf{u}_{h}-\hat{\mathbf{u}}\right) \mathrm{d} \Omega, \quad\|u\|^{2}=\int_{\Omega} \hat{\mathbf{u}} \cdot \hat{\mathbf{u}} \mathrm{d} \Omega \\
\left\|e_{\varepsilon}\right\|^{2} & =\int_{\Omega} \mathbf{D}^{-1}\left(\boldsymbol{\sigma}_{h}-\hat{\boldsymbol{\sigma}}\right) \cdot\left(\boldsymbol{\sigma}_{h}-\hat{\boldsymbol{\sigma}}\right) \mathrm{d} \Omega, \quad\|\varepsilon\|^{2}=\int_{\Omega} \mathbf{D}^{-1} \hat{\boldsymbol{\sigma}} \cdot \hat{\boldsymbol{\sigma}} \mathrm{d} \Omega
\end{aligned}
$$

where $\mathbf{D}$ is the elasticity tensor, $\boldsymbol{\sigma}_{h}$ and $\mathbf{u}_{h}$ denote respectively stress tensor and displacement vector relevant to an approximation (based on an $h$-scaled domain discretization) of the exact solution $\hat{\boldsymbol{\sigma}}, \hat{\mathbf{u}}$. Evidently, this latter is not known a priori for problems under investigation. Nevertheless, estimates of relative energy error can be performed following the procedure proposed by Zienkiewicz and Zhu [59] and available within the Ansys tool. Being convergence in linear elasticity ensured by general theorems, this approach is based on the assumption that, for a given $h$-scaled discretization, the exact stress $\hat{\sigma}$ in a point can be approximated with the averaged field $\bar{\sigma}_{h}$, computed in an $h$-scaled neighborhood of that point through the numerical solution $\boldsymbol{\sigma}_{h}$. Referring to material properties summarized in Table 2 and considering the previously-introduced mesh refinement levels, Tables 3 and 4 indicate energy error estimates computed at each interfacial domain $\Omega_{\mathrm{t}}$ and $\Omega_{\mathrm{c}}$ (see Fig. 4) of all the present finite-element models. When mesh parameters $h_{\mathrm{o}}$ and $h_{i}$ reduce, a convergent behaviour clearly appears and the best accuracy is experienced with M4 meshes. Accordingly, M4-based displacement solution $\mathbf{u}_{\mathrm{M} 4}$ can be assumed as a suitable approximation of the exact field $\hat{\mathbf{u}}$. Therefore, relative displacement error (4) can be estimated as:

$$
E_{u} \approx \frac{\sum_{k}\left[\mathbf{u}_{h}\left(N_{k}\right)-\mathbf{u}_{M 4}\left(N_{k}\right)\right] \cdot\left[\mathbf{u}_{h}\left(N_{k}\right)-\mathbf{u}_{M 4}\left(N_{k}\right)\right]}{\sum_{k} \mathbf{u}_{M 4}\left(N_{k}\right) \cdot \mathbf{u}_{M 4}\left(N_{k}\right)}
$$

where sums are performed on nodes $N_{k}$ belonging to interfacial bone region $\Omega_{\mathrm{t}} \cup \Omega_{\mathrm{c}}$ and notation $f\left(N_{k}\right)$ denotes value of $f$ at

\begin{tabular}{|c|c|c|c|c|c|c|c|c|c|c|}
\hline & ITI 1 & & ITI 2 & & BR 1 & & BR 2 & & ANK & \\
\hline Mandibular & $\Omega_{\mathrm{c}}$ & $\Omega_{\mathrm{t}}$ & $\Omega_{\mathrm{c}}$ & $\Omega_{\mathrm{t}}$ & $\Omega_{\mathrm{c}}$ & $\Omega_{\mathrm{t}}$ & $\Omega_{\mathrm{c}}$ & $\Omega_{\mathrm{t}}$ & $\Omega_{\mathrm{c}}$ & $\Omega_{\mathrm{t}}$ \\
\hline M1 & 59.2 & 60.2 & 55.4 & 83.2 & 63.2 & 59.5 & 66.9 & 58.5 & 71.2 & 65.3 \\
\hline M2 & 32.6 & 37.6 & 32.7 & 40.2 & 35.9 & 40.3 & 33.2 & 37.3 & 42.9 & 37.2 \\
\hline M3 & 17.2 & 21.4 & 17.1 & 21.4 & 19.7 & 21.4 & 19.2 & 19.1 & 21.4 & 18.4 \\
\hline M4 & 9.6 & 11.5 & 8.9 & 13.1 & 10.1 & 12.3 & 10.6 & 11.2 & 12.1 & 11.4 \\
\hline Maxillary & $\Omega_{\mathrm{c}}$ & $\Omega_{\mathrm{t}}$ & $\Omega_{\mathrm{c}}$ & $\Omega_{\mathrm{t}}$ & $\Omega_{\mathrm{c}}$ & $\Omega_{\mathrm{t}}$ & $\Omega_{\mathrm{c}}$ & $\Omega_{\mathrm{t}}$ & $\Omega_{\mathrm{c}}$ & $\Omega_{\mathrm{t}}$ \\
\hline M1 & 71.3 & 73.2 & 72.7 & 78.4 & 68.7 & 70.3 & 71.0 & 65.5 & 73.2 & 74.6 \\
\hline M2 & 40.7 & 42.5 & 39.4 & 43.6 & 39.8 & 45.7 & 41.2 & 40.2 & 44.7 & 40.9 \\
\hline M3 & 22.6 & 25.6 & 21.3 & 22.5 & 23.7 & 25.4 & 23.5 & 21.8 & 22.4 & 21.6 \\
\hline M4 & 15.3 & 17.5 & 10.2 & 14.1 & 12.4 & 15.6 & 13.4 & 13.9 & 12.9 & 14.2 \\
\hline
\end{tabular}
the coordinates of $N_{k}$. Fig. 5 shows the relative displacement error estimate $E_{u}$ versus the mesh parameter $h_{i}$ for all the ana-

Table 3

Relative energy norm error estimate $E_{\varepsilon}(\%)$ versus mesh refinement level for single-tooth restoration models based on commercial implants 
Table 4

Relative energy norm error estimate $E_{\varepsilon}(\%)$ versus mesh refinement level for restoration models based on double-implant systems

\begin{tabular}{|c|c|c|c|c|c|c|}
\hline & $\operatorname{DIRB}(\mathrm{A})$ & & $\mathrm{MI}_{0}$ & & $\mathrm{MI}_{25}$ & \\
\hline Mesh & $\Omega_{\mathrm{c}}$ & $\Omega_{\mathrm{t}}$ & $\Omega_{\mathrm{c}}$ & $\Omega_{\mathrm{t}}$ & $\Omega_{\mathrm{c}}$ & $\Omega_{\mathrm{t}}$ \\
\hline M1 & 68.4 & 78.3 & 75.1 & 77.2 & 73.2 & 80.3 \\
\hline M2 & 40.2 & 41.8 & 45.6 & 47.4 & 40.8 & 44.8 \\
\hline M3 & 20.5 & 22.3 & 22.7 & 25.1 & 23.4 & 28.7 \\
\hline M4 & 11.8 & 12.4 & 15.3 & 16.4 & 14.3 & 17.2 \\
\hline
\end{tabular}
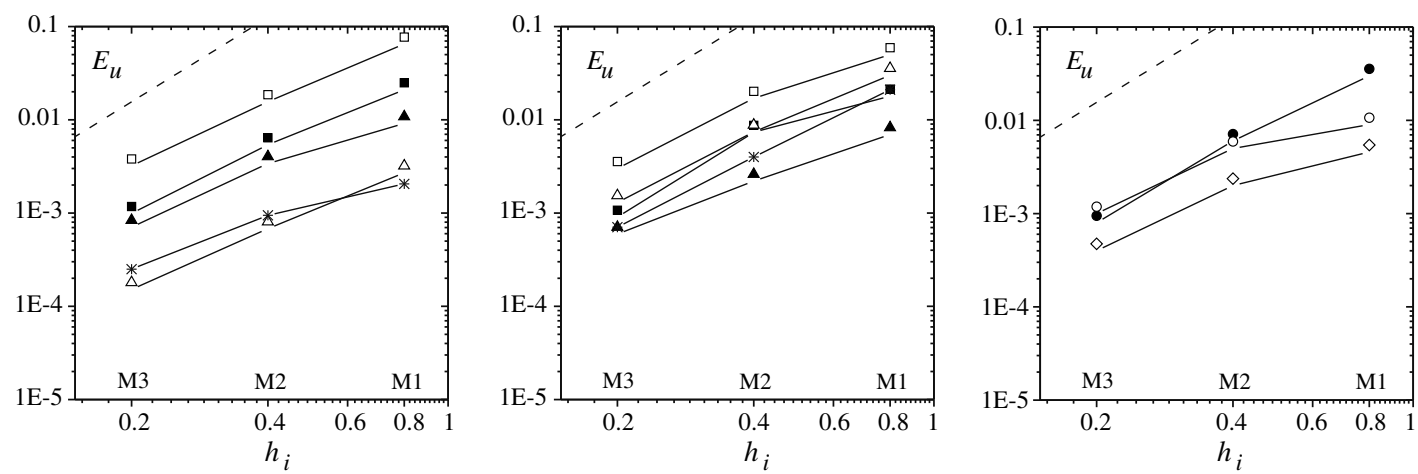

Fig. 5. Relative displacement error $E_{u}$ versus the mesh parameter $h_{i}$ for all the analyzed models. $E_{u}$ is estimated through the Eq. (7), approximating the exact displacement field by the M4-based numerical solution. From the left: mandibular, maxillary and multi-implant applications. ( $\square$ ) ITI 1 ; ( $\square$ ) ITI 2; ( $\boldsymbol{\Delta}$ ) Branemark 1; $(\triangle)$ Branemark 2; $(*)$ Ankylos; $(\diamond)$ DIRB (load position A); $(\bullet) \mathrm{MI}_{0}$; (०) $\mathrm{MI}_{25}$; (---) maximum theoretical convergence slope [58].

lyzed models, when material properties of Table 2 are employed. Comparison of numerical results (meshes are not uniform) with the maximum theoretical convergence rate [58] (dashed line in Fig. 5) highlights good convergence behaviours.

It should be marked out that percent errors in energy norm relevant to M4-based models remain quite high (about 9$18 \%$ ), the most of energy error being located at screw threads, where certain point-wise inaccuracies are expected as a consequence of geometrical singularities. Nevertheless, analysis of Fig. 5 proves that displacement results at bone-implant interface are very accurate. Accordingly, averaged stress fields $\overline{\boldsymbol{\sigma}}_{\mathrm{M} 4}$ can be expected themselves accurate and M4-based meshes can be considered effective for giving suitable quantitative approximations of real stress distributions at peri-implant regions.

\subsection{Single-tooth commercial implants}

Figs. 6 and 7 show Von Mises stress distributions relevant to single-tooth applications based on the five commercial endosteal dental implants here investigated. In detail, with reference to the cross-section at $y=0$, stress contours on both maxillary and mandibular bone segments are put in comparison. In order to give significant indications at both compact and trabecular peri-implant regions, two different contour legends are used.

Under the assumption of a complete osseous integration, stress concentration areas are localized around implant neck at the cortical bone interface. According to the geometrical properties summarized in Table 1, dimensions of cortical bone regions probably affected by overloading and high stress values seem to be influenced by implant diameter $d$, irrespective of bone-implant interface length $\ell$. Nevertheless, when $\ell$ increases for a given $d$ more homogeneous stress distributions and reduced stress concentrations at cancellous bone are obtained. Moreover, although implants Branemark 1 and Ankylos have comparable values of $d$, cortical bone shape around Ankylos device (due to its platform-switching configuration) induces lower stress values at peri-implant compact bone.

These considerations are fully confirmed by the analysis of Fig. 8, showing principal and Von Mises stress measures at bone-implant interface (cortical and trabecular) for insertions in both mandibular and maxillary molar segments. Proposed results highlight that for compact bone the highest values of Von Mises $\left(\sigma_{\mathrm{v}}\right)$ and compressive $\left(\sigma_{\mathrm{C}}\right)$ stresses arise in maxillary segment ( $\sigma_{\mathrm{v}}^{\mathrm{c}}$ ranging from about $65 \mathrm{MPa}$-Ankylos - to $220 \mathrm{MPa}$ - ITI 2 -; $\sigma_{\mathrm{c}}^{\mathrm{c}}$ from about $36 \mathrm{MPa}$ - Ankylos - to about $375 \mathrm{MPa}$ - ITI 2 -) and they are deeply dependent on implant shape. On the other hand, tensile stress peaks are significantly lower (ranging from about $18 \mathrm{MPa}$ to about $100 \mathrm{MPa}$ ) than compressive ones and their values seems to be weakly affected by implant geometry.

Quantitative stress analysis highlights that Ankylos implant exhibits the best performance at cortical bone interface, both in mandibular and maxillary placement, leading to the lowest values of the cortical risk indicators and inducing at the same time fully acceptable stresses at trabecular bone interface (at the most equal to $4 \mathrm{MPa}$ ). On the other hand, the worst load transfer mechanism is numerically experienced on mandibular (maxillary) segment when implants Branemark 1 and ITI 2 


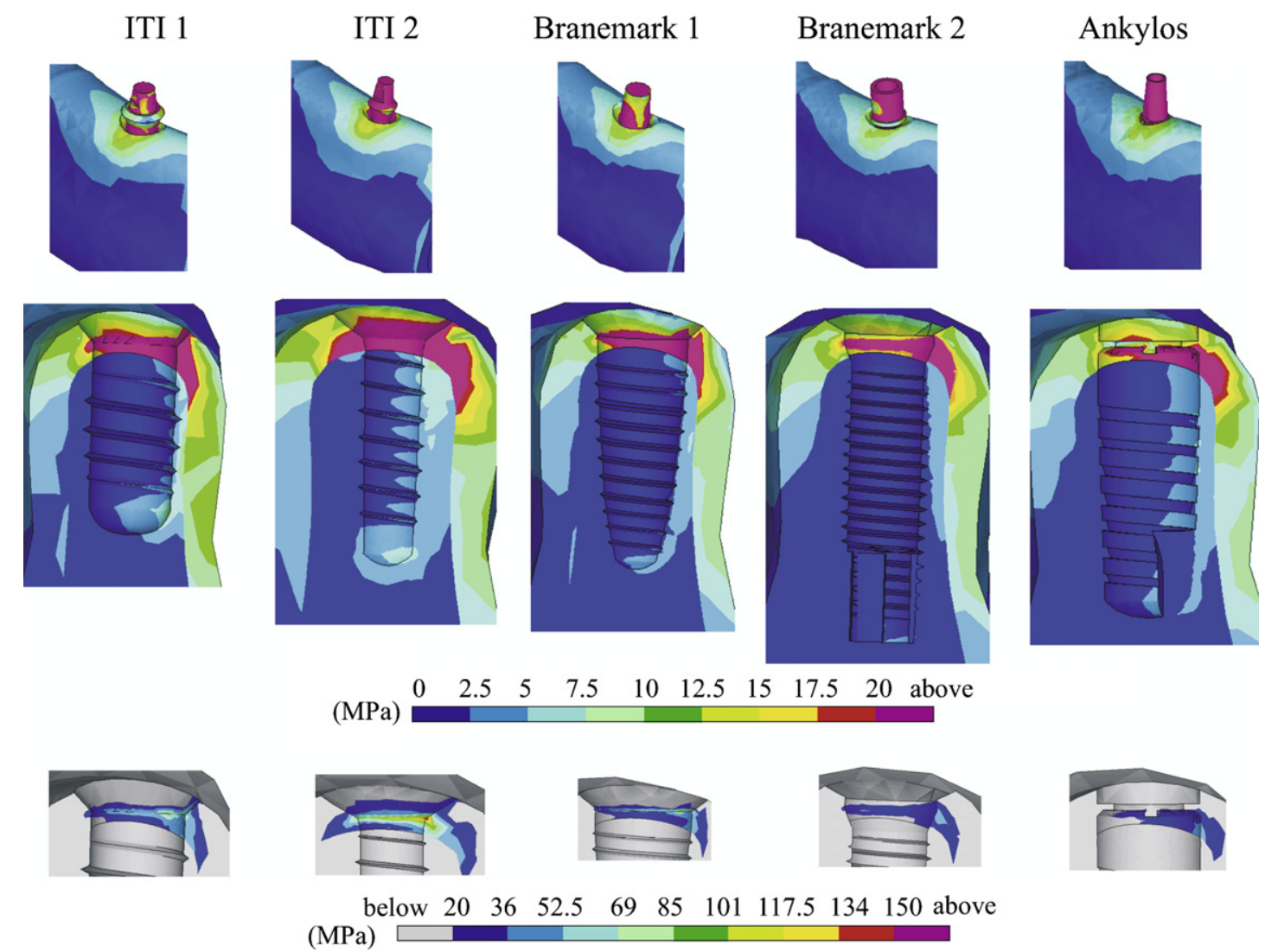

Fig. 6. Von Mises stress contours on the cross-section at $y=0$ for single-tooth commercial endosteal implants in molar mandibular segment.

(ITI 2) are considered. In detail, stress measures in mandibular cortical bone and relevant to Branemark 1 implant overcome by about $140 \%$ in tension and $290 \%$ in compression ( $180 \%$ considering $\sigma_{\mathrm{v}}$ ) those of Ankylos system. Moreover, when ITI 2 implant is experienced stress values in maxillary cortical bone are much greater (by about $150 \%$ in tension, $600 \%$ in compression, $300 \%$ for the Von Mises measure) than Ankylos-implant's ones. On compact bone the previously-introduced physiological limits [39,41] are exceeded on maxillary segment in compression when implants ITI 1, ITI 2 and Branemark 2 are considered, whereas tensile bone strength is never reached.

As far as overloading risk indicators at cancellous bone are concerned, it can be observed that tensile peaks are always greater than compressive ones and significant concentrations can appear at trabecular-compact interface as well as, with smaller values, at the implant bottom region. Strength of cancellous bone (about $5 \mathrm{MPa}$ [39]) is exceeded, mainly in tension, at the concentration areas for all the investigated implants except for Ankylos.

\subsection{Influence of trabecular bone quality}

In order to analyze influence of trabecular bone quality on load transfer mechanisms, trabecular Young's modulus $E_{\mathrm{t}}$ is varied as a parameter (see Section 2.2). Two commercial single-tooth implants characterized by comparable values of diameter $d$ (Branemark 1 and Ankylos) are analyzed considering mandibular applications.

Fig. 9 depicts Von Mises stress distributions on the cross-section at $y=0$ for different values of $E_{\mathrm{t}}$. It clearly appears that when trabecular bone quality increases (i.e., $E_{\mathrm{t}}$ increases) more homogeneous stress contours are experienced, resulting in a reduction of stress peaks at cortical bone region close to the implant neck. These considerations are confirmed by analyzing Fig. 10, which summarizes the highest and average values of stress measures (1)-(3) at peri-implant interface.

In detail, varying $E_{\mathrm{t}}$ from $0.5 \mathrm{GPa}$ to $9.5 \mathrm{GPa}$, the highest values of Von Mises and compressive stress measures on cortical bone reduce by about 54\% and 40\% (51\% and 49\%), respectively, when Branemark 1 (Ankylos) implant are considered, whereas tensile stresses increase by about $62 \%$ for Branemark 1 implant and decrease by about $35 \%$ for Ankylos device. Moreover, as far as trabecular bone is concerned, a better cancellous bone quality leads to greater stress values, resulting in a reduction of stress gradients at compact-cancellous interface. Finally, it can be observed that Ankylos implant, due to platform-switching configuration, exhibits the best performance on compact bone for all the values of $E_{\mathrm{t}}$ here considered, whereas at cancellous implant-bone interface implants Ankylos and Branemrak 1 are practically comparable in terms of 

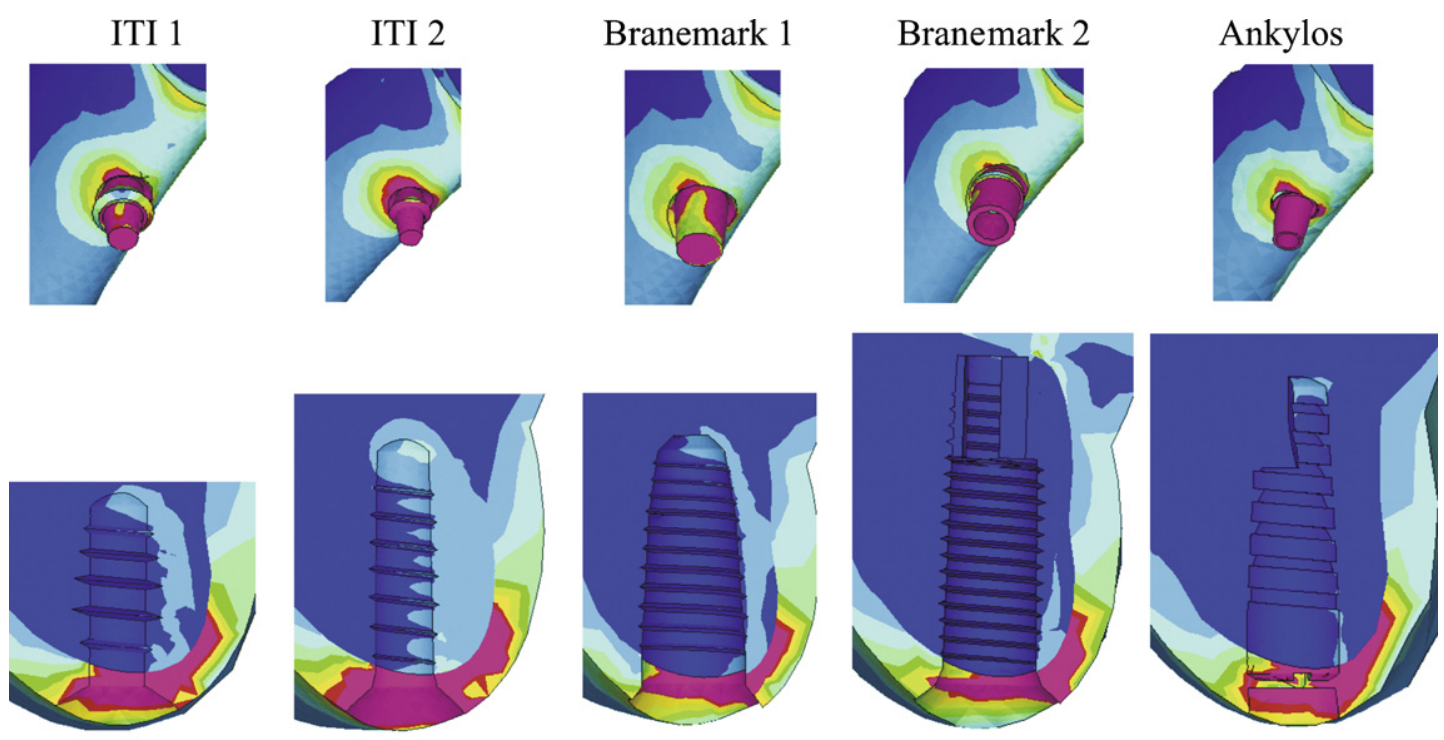

$\begin{array}{llllllllll}0 & 2.5 & 5 & 7.5 & 10 & 12.5 & 15 & 17.5 & 20 & \text { above }\end{array}$
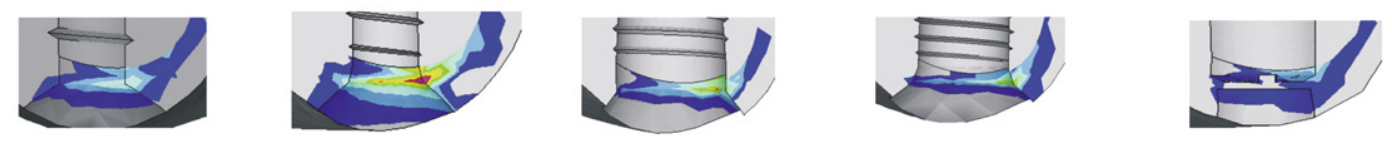

below $\begin{array}{lllllllll}20 & 36 & 52.5 & 69 & 85 & 101 & 117.5 & 134 & 150 \\ \text { above }\end{array}$

\section{(MPa)}

Fig. 7. Von Mises stress contours on the cross-section at $y=0$ for single-tooth commercial endosteal implants in molar maxillary segment.

average stress values. Nevertheless, trabecular risk indicators strongly reduce when implant-bone interface length $\ell$ increases, that is passing from $\ell=9 \mathrm{~mm}$ (Branemark 1 ) to $\ell=11 \mathrm{~mm}$ (Ankylos).

\subsection{Double-implant applications}

Figs. 11 and 12 show Von Mises stress distributions on the mesio-distal cross-section at $x=0$ and relevant to numerical simulations performed on double-implant systems placed into the mandibular bone segment. Fig. 11 refers to DIRB device, whereas Fig. 12 is relevant to non-conventional mini-implant applications (vertical $-\mathrm{MI}_{0}$ - and angled $-\mathrm{MI}_{25}$-, see Fig. 2). Moreover, Fig. 13 summarizes the highest and average values of stress measures (1)-(3) computed at trabecular and cortical peri-implant interface.

As far as DIRB system is concerned, three different occlusal force locations are considered: at the retaining bar mid-span (A) and in correspondence of mesial (B) and distal (C) Ankylos implants (see Fig. 2b). It can be observed that stress values computed in the case B at mesial implant-bone interface are fully comparable with stress values at distal region when case $\mathrm{C}$ is considered. Moreover, analysis of stress measures computed in the case A confirms that a middle-located occlusal force induces similar stress levels at both mesial and distal bone interfaces. When the case of a not-centered load is investigated (cases B and C) and with respect to stress levels relevant to the case A, stress measures increase by about $50 \%$ at bone interface close to the force location and reduce of the same percentage at the opposite side. It is worth noting that tensile and compressive physiological limits are practically never exceeded, resulting in a good mechanical performance of this Ankylos-based multi-implant device.

As far as non-conventional mini-implant systems are concerned, the highest values of principal stress (Von Mises) measures on cortical bone are computed in the case of the angled device $\mathrm{MI}_{25}\left(\mathrm{MI}_{0}\right)$. In detail, compressive and tensile risk indicators relevant to $\mathrm{MI}_{25}$ are greater than those experienced for $\mathrm{MI}_{0}$ by about $90 \%$. Moreover, although average stresses are fully acceptable in a physiological sense, cortical peaks slightly exceed bone strength in tension when $\mathrm{MI}_{25}$ device is experienced. On the other hand, reduced length of mini-fixtures leads to trabecular stress values greater than those of standard dental implants and the relating stress peaks could locally exceed physiological limits for both MI cases. Nevertheless, proposed numerical results show that MI devices exhibit a fully comparable or even better mechanical behaviour than some standard commercial single-tooth implants, such as the Branemark's or the ITI's analyzed in this study. Furthermore, it should be taken into account that the small inter-axis length between mini-fixtures should induce, as a consequence of 

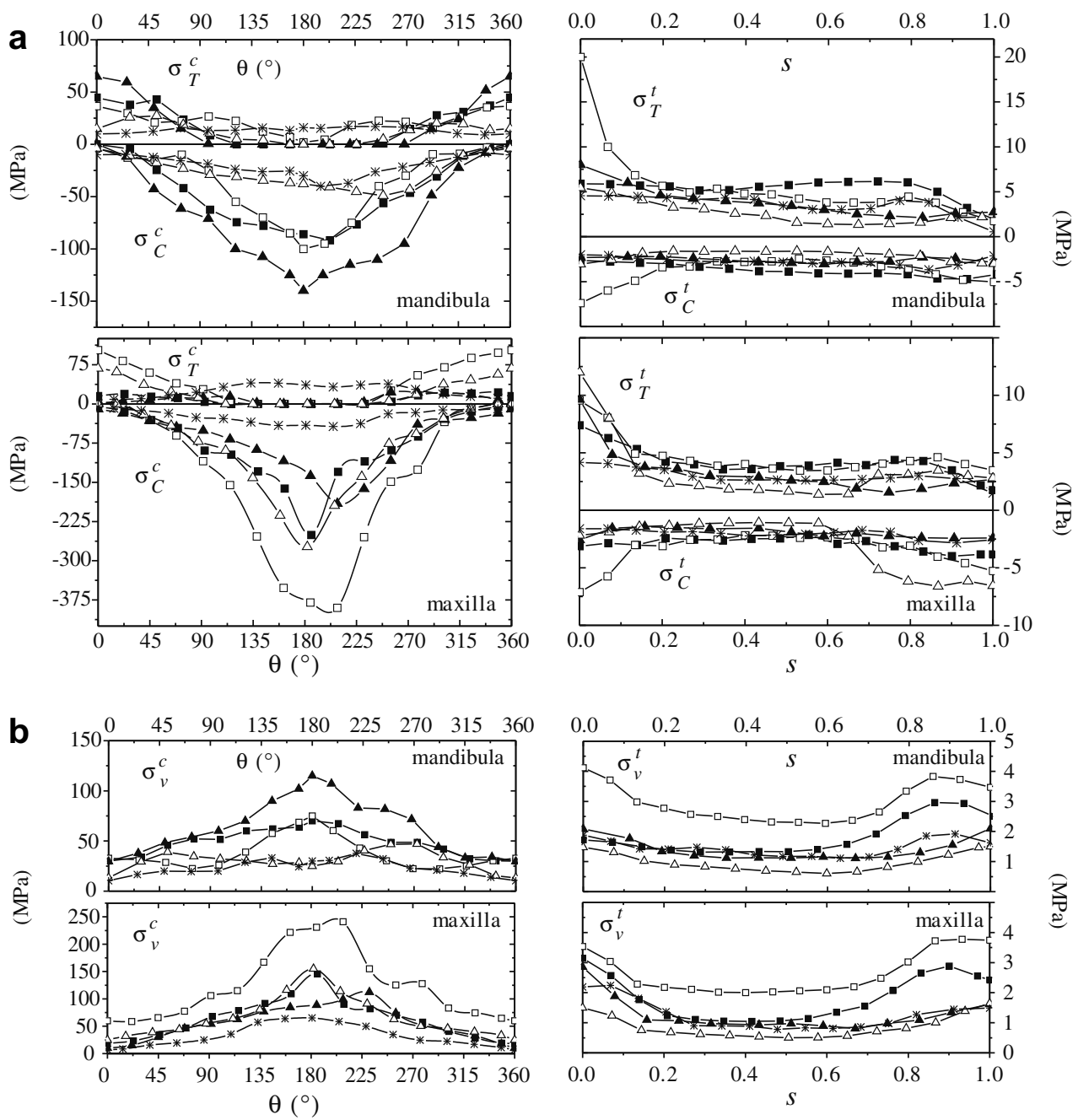

Fig. 8. (a) Principal $\left(\sigma_{\mathrm{C}}, \sigma_{\mathrm{T}}\right)$ and (b) Von Mises $\left(\sigma_{\mathrm{v}}\right)$ stress measures computed at compact ( $\sigma^{\mathrm{c}}$, on the left) and trabecular $\left(\sigma^{\mathrm{t}}\right.$, on the right) peri-implant interface for mandibular and maxillary insertions. Symbol $s$ denotes dimensionless abscissa along implant axis, such that $s=0$ at cortical-trabecular bone interface and $s=1$ at inserted implant end. ( $\mathbf{\square})$ ITI 1; ( $\square)$ ITI 2; $(\boldsymbol{\Delta})$ Branemark 1; $(\Delta)$ Branemark 2; (*) Ankylos.

the osseous integration process, a thicker cortical layer, resulting in a better load transfer mechanisms and also in long-term stability advantages.

\section{Discussion and concluding remarks}

In this paper five commercially-available osseointegrated dental implants (two Straumann devices, two Nobel Biocare implants and a Dentsply Friadent one) as well as a number of multiple-implant applications have been numerically investigated by means of static linearly elastic three-dimensional finite-element analyses, under functional loads and considering insertions in both mandibular and maxillary molar bone segments.

Three-dimensional numerical models have been built-up employing CT images and comparative techniques. Full osseous integration and different levels of trabecular bone quality have been taken into account. Moreover, depending on crestal bone loss induced by implant shape, different compact bone geometries around implant neck have been modelled. Stress analyses have been performed both in terms of global and local (at bone-implant interface) stress measures, in order to investigate about influence of fixture shape on implant mechanical performance and to give quantitative indications relevant to the risk of bone weakening or loss due to local tissue overloading. Results of a preliminary convergence analysis have been also presented, validating effectiveness and accuracy of proposed finite-element models.

Within the limitations of this study, the numerically-analyzed five commercial osseointegrated implants exhibit deeply different mechanical behaviour depending on their shape parameters and on the site of placement. In detail, due to different bone segment geometries as well as to different bone mechanical properties, finite element results show as, for a given im- 

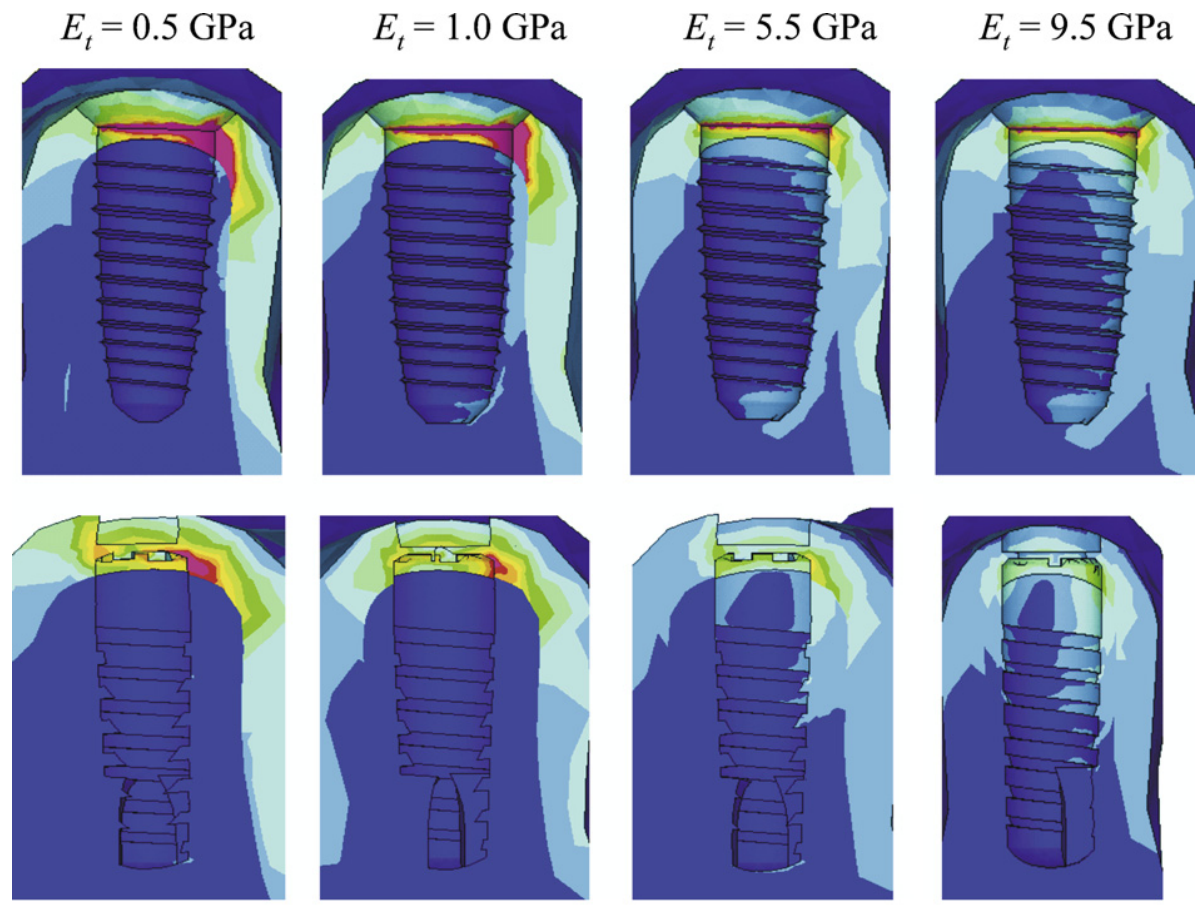

$\begin{array}{lllllllll}0 & 3.7 & 8.4 & 11.2 & 15 & 18.7 & 22.5 & 26.2 & 30\end{array}$

(MPa)

Fig. 9. Von Mises stress contours on the cross-section at $y=0$ for Branemark 1 (first row) and Ankylos (second row) implants in mandibular segment versus trabecular Young's modulus $E_{\mathrm{t}}$.
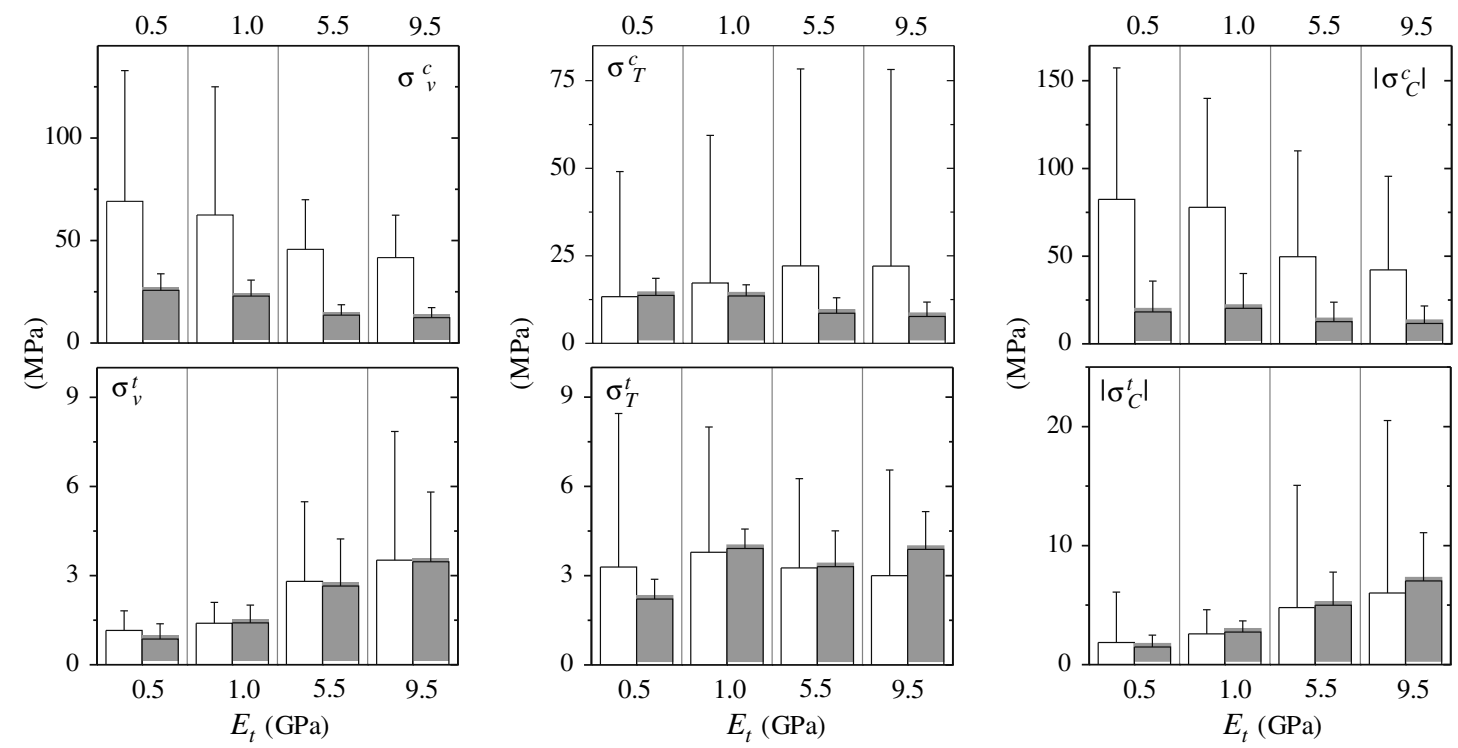

Fig. 10. Stress measures computed at compact ( $\sigma^{\mathrm{c}}$, first row) and trabecular ( $\sigma^{\mathrm{t}}$, second row) peri-implant interface versus trabecular Young's modulus $E_{\mathrm{t}}$. Bars denote average values, lines indicate maximum values. Branemark 1 (white bars) and Ankylos (gray bars) implants in mandibular segment.

plant, stress distributions on mandibular and maxillary bone segments can be deeply dissimilar, resulting in higher stress concentrations in maxillary bone than mandibular tissues. Accordingly, proposed quantitative stress analyses may be considered as a contribution for understanding clinical evidences stating that maxillary implants can be affected by a greater failure percentage than mandibular ones [17,18,28,33,47]. 
a

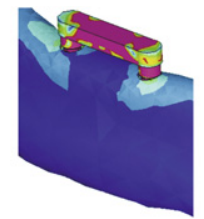

b

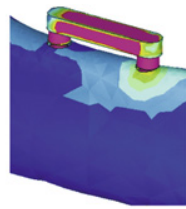

C
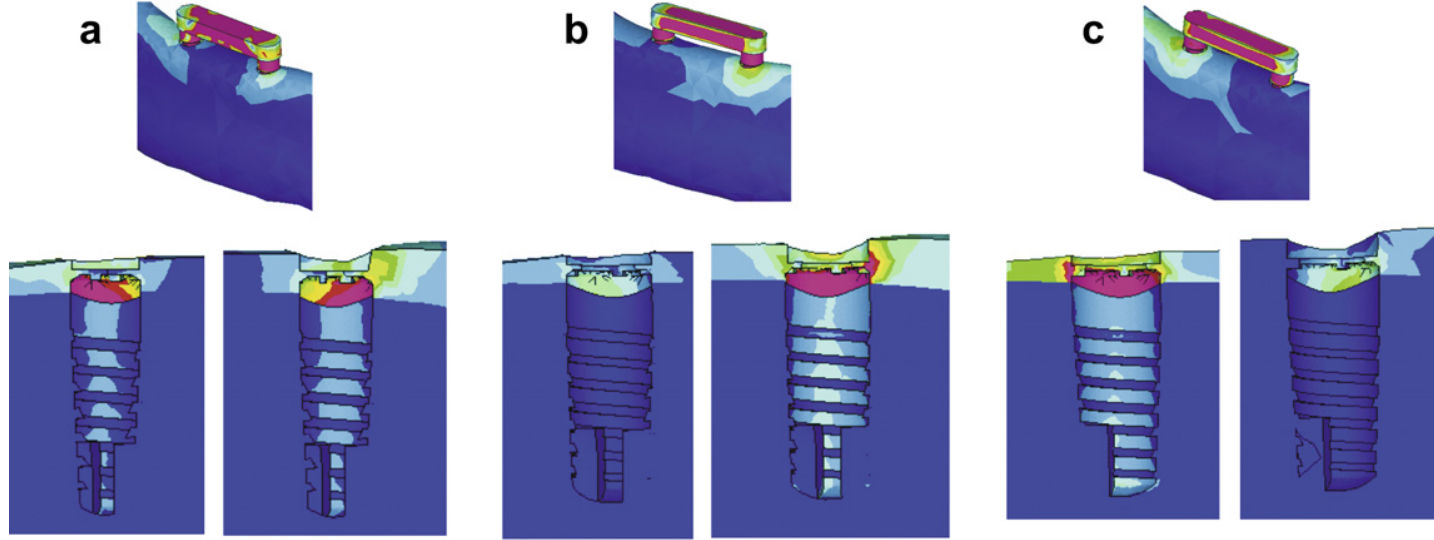

(MPa)

$\begin{array}{llllllllll}0 & 2.5 & 5 & 7.5 & 10 & 12.5 & 15 & 17.5 & 20 & \text { above }\end{array}$
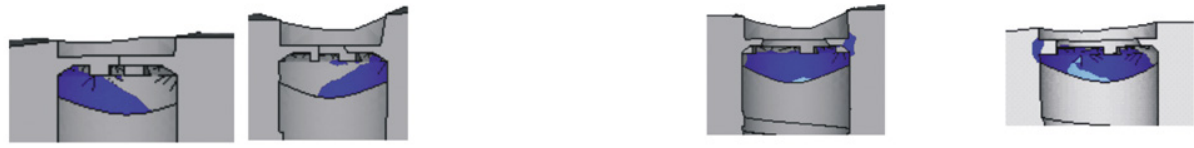

below $\begin{array}{llllllllll}20 & 36 & 52.5 & 69 & 85 & 101 & 117.5 & 134 & 150 & \text { above }\end{array}$

(MPa) $\begin{array}{rllllllll}\square \\ \square\end{array}$

Fig. 11. Von Mises stress contours on the cross-section at $x=0$ for DIRB application and considering different load locations: (a) at the retaining bar midspan; (b) at the mesial implant; (c) at the distal implant.
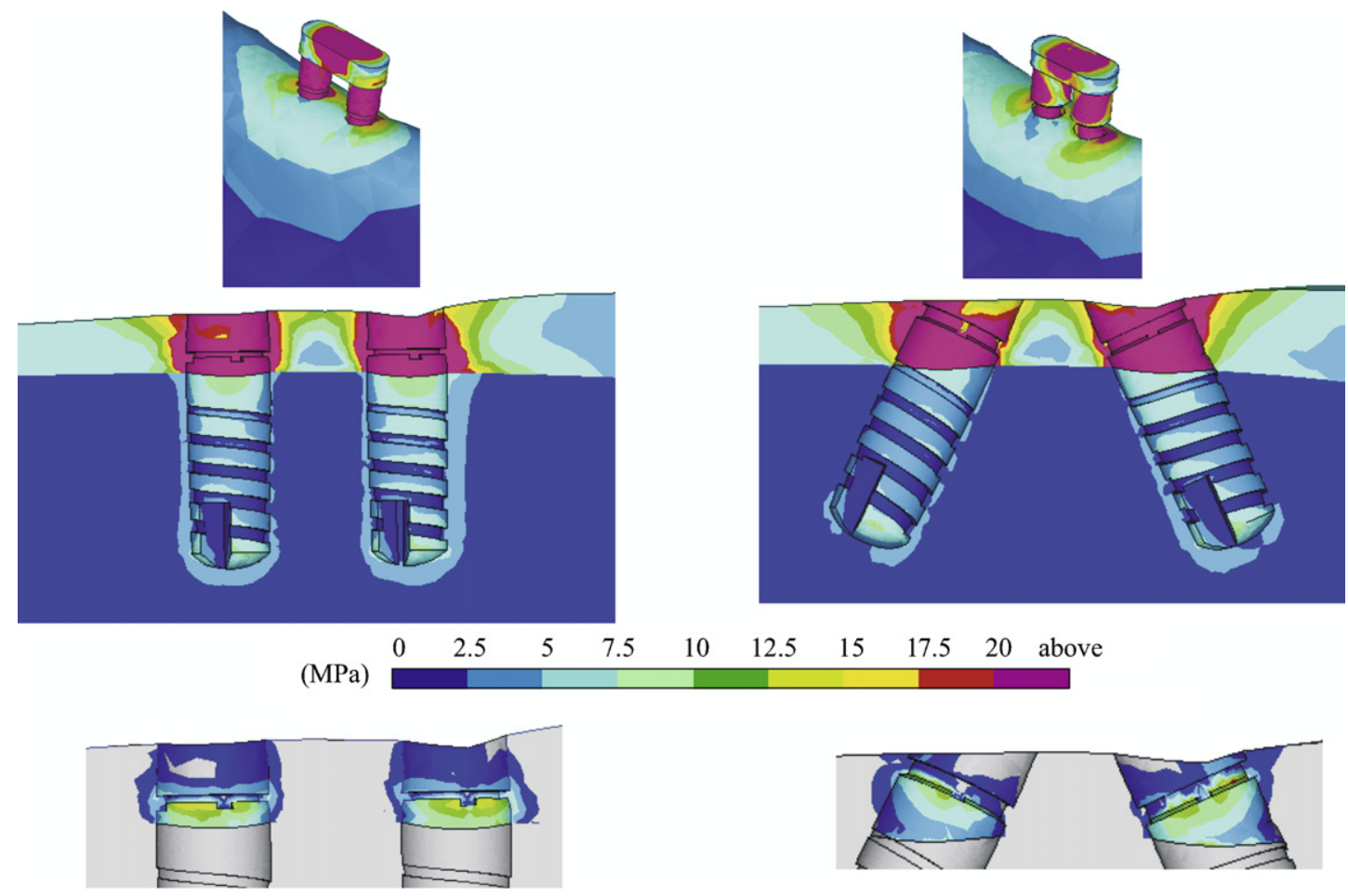

(MPa)

Fig. 12. Von Mises stress contours on the cross-section at $x=0$ for non-conventional mini-implant applications: $\mathrm{MI}_{0}$ on the left and $\mathrm{MI}_{25}$ on the right. 


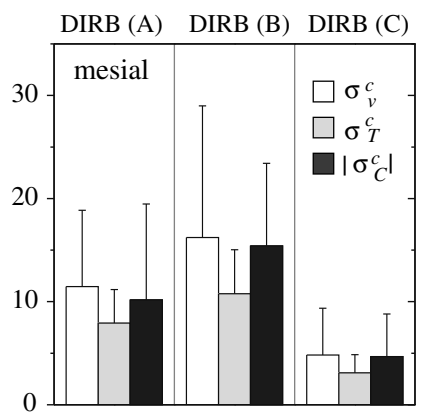

0

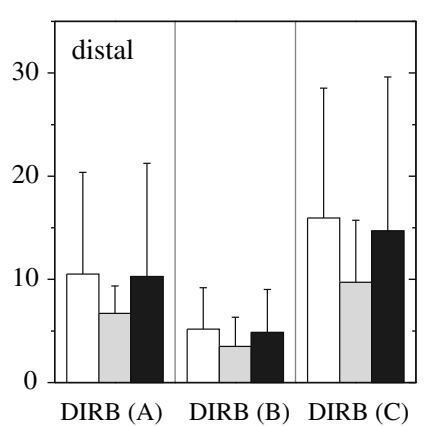

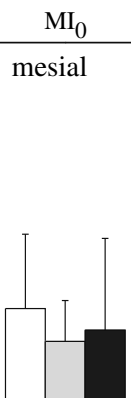

distal
$\mathrm{MI}_{25}$
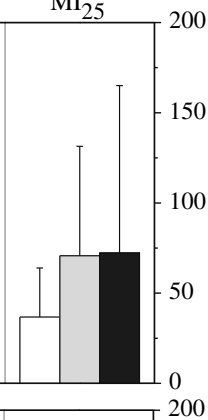

DIRB (A) DIRB (B) DIRB (C)

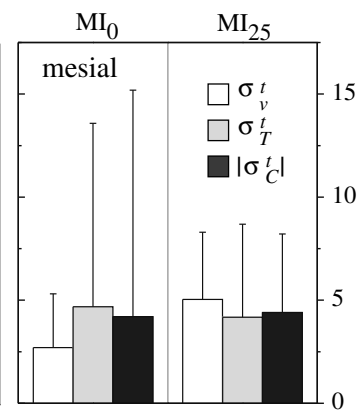

0

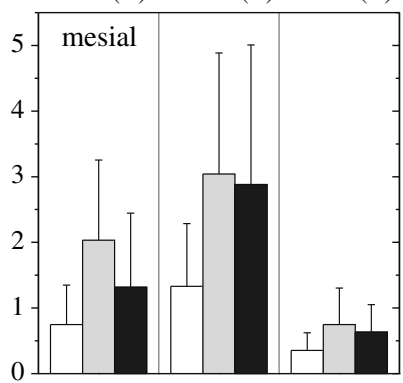

50
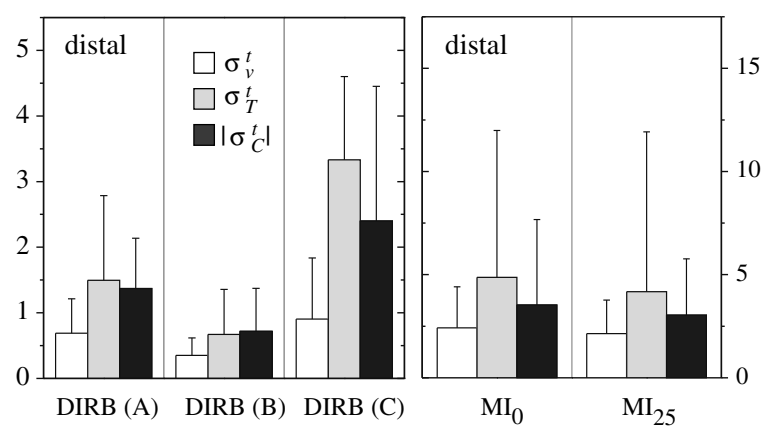

Fig. 13. Stress measures (in MPa) computed at compact ( $\sigma^{\mathrm{c}}$, on the left) and trabecular ( $\sigma^{\mathrm{t}}$, on the right) peri-implant interface for double-implant devices. First row refers to mesial implant, and second row to distal implant. Bars denote average values, lines indicate maximum values.

Simulation results highlight also the influence on load transfer mechanisms of implant's length and diameter as well as of crestal bone loss and platform switching configurations. In agreement with the trend numerically experienced by Bozkaya et al. [5], Himmlová et al. [23] and Holmgren et al. [24], maximum implant diameter seems to affect stress peaks at cortical bone but not at the trabecular one, whereas stress values and their distributions at cancellous bone-implant interface are mainly affected by implant interface length. Nevertheless, proposed results show that implant diameter is more important for more homogeneous and efficient stress distributions than implant length, confirming that bone overloading risk essentially affects regions around implant neck.

Comparisons of numerical results relevant to implants with similar maximum diameters and bone-implant interface lengths have also indicated that stress peaks strongly reduce if platform switching configurations (e.g., Ankylos implant) are considered. Accordingly, under a conventional bucco-lingual intrusive occlusal load, the reduced crestal bone loss due to platform switching allows to obtain the best performance in terms of stress distributions at compact bone interface, together with fully acceptable stress values at cancellous bone. On the other hand, when significant crestal bone loss is modelled, physiological strength limits could be exceeded at both trabecular and compact bone, inducing implant failure. These results are qualitatively in agreement with those obtained by Bozkaya et al. [5] and by Maeda et al. [37], highlighting as possible overloading at compact bone occurs in compression, whereas at the interface between cortical and trabecular bone overloading can occur in tension.

The influence of trabecular bone quality has been also analyzed. In detail, when trabecular Young's modulus increases more homogeneous stress distributions have been computed, resulting in a reduction of both cortical stress peaks and cancellous-compact stress gradients.

As far as double-implant applications are concerned, analysis of DIRB system shows the effectiveness of this device when commercial Ankylos implants are employed. As a matter of fact, quantitative stress analysis relevant to different loading locations upon the retaining bar highlights that compressive and tensile bone physiological limits are not exceeded. On the other hand, overloading states appear in single-tooth mini-implant applications (MI) based on two Ankylos-type mini-fixtures. Nevertheless, their load transmission mechanisms are fully comparable with those of a number of conventional single-tooth implants. Accordingly, MI systems can be considered as a concrete alternative to traditional single-tooth implants, when geometrical configuration of the site of placement and bone quantity and quality (particularly in sinus zone) do not allow to employ a single greater fixture. Moreover, mini-implant devices should allow more effective long-term stability results, especially when angled mini-implant configurations are experienced.

In this study, even if different crestal bone loss geometries have been taken into account, full osseous integration has been assumed between implants and bone. Clinically, this may be considered as an optimistic assumption. Moreover, concentrated static loads have been analyzed, whereas real conditions during mastication are represented by time-dependent distributed forces. Nevertheless, observing that bone remodelling is beyond the scope of this investigation, and as confirmed by a number of well-established numerical results, these assumptions can be considered reasonable and convenient, in a 
computational sense, for deducing significant and clinically-useful indications. Accordingly, proposed fully three-dimensional simulation approach can be considered as an accurate and effective tool for performing stress-based performance evaluation of osseointegrated dental implants.

\section{Acknowledgements}

This work has been developed within the framework of Lagrange Laboratory, an European research group comprising CNRS, CNR, the Universities of Rome "Tor Vergata", Calabria, Cassino, Pavia, and Salerno, Ecole Polytechnique, University of Montpellier II, ENPC, LCPC, and ENTPE.

\section{References}

[1] K. Arkça, H. İplikçioglu, Comparative evaluation of the effect of diameter, length and number of implants supporting three-unit fixed partial prostheses on stress distribution in bone, J. Dent. 30 (2002) 40-46.

[2] E. Asmussen, A. Peutzfeldt, A. Sahafi, Finite element analysis of stresses in endodontically treated, dowel-restored teeth, J. Prosthet. Dent. 94 (2005) 321-329.

[3] P. Ausiello, A. Apicella, C.L. Davidson, Effect of adhesive layer properties on stress distribution in composite restorations: a 3D finite element analysis, Dent. Mater. 18 (2002) 295-303.

[4] C.M. Becker, D.A. Kaiser, Implant-retained cantilever fixed prosthesis: where and when, J. Prosthet. Dent. 84 (2000) 432-435.

[5] D. Bozkaya, S. Muftu, A. Muftu, Evaluation of load transfer characteristics of five different implants in compact bone at different load levels by finite elements analysis, J. Prosthet. Dent. 92 (2004) 523-530.

[6] J.B. Brunski, Biomechanics of dental implants, in: M. Block, J.N. Kent, L.R. Guerra (Eds.), Implants in Dentistry, W.B. Saunders, Philadelphia, 1997, pp. 63-71.

[7] D.P. Callan, A. O' Mahony, C.M. Cobb, Loss of crestal bone around dental implants: a retrospective study, Implant Dent. 7 (1998) $258-266$.

[8] D.R. Carter, M.C. Van Der Meulen, G.S. Beaupre, Mechanical factors in bone growth and development, Bone 18 (1996) S5-S10.

[9] F. Chen, K. Terada, K. Hanada, I. Saito, Anchorage effects of a palatal osseointegrated implant with different fixation: a finite element study, Angle Orthod. 75 (2005) 593-601.

[10] H.J. Chun, S.Y. Cheong, J.H. Han, S.J. Heo, J.P. Chung, I.C. Rhyu, et al, Evaluation of design parameters of osseointegrated dental implants using finite element analysis, J. Oral Rehabil. 29 (2002) 565-574.

[11] H.J. Chun, D.N. Park, C.H. Han, S.J. Heo, M.S. Heo, J.Y. Koak, Stress distributions in maxillary bone surrounding dental implants with different overdenture attachments, J. Oral Rehabil. 32 (2005) 193-205.

[12] H.J. Chun, H.S. Shin, C.H. Han, S.H. Lee, Influence of implant abutment type on stress distribution in bone under various loading conditions using finite element analysis, Int. J. Oral Maxillofac. Implants 21 (2006) 195-202.

[13] L. Chun-Li, K. Yu-Chan, L. Ting-Sheng, Effects of dental implant length and bone quality on biomechanical responses in bone around implants: a 3D non-linear finite element analysis, Biomed. Eng. Appl. Basis Comm. 17 (2005) 44-49.

[14] D.L. Cochran, A comparison of endosseous dental implant surfaces, J. Periodontol. 70 (1999) 1523-1539.

[15] S.C. Cowin, Bone Mechanics Handbook, CRC Press, Boca Raton, 2001.

[16] M. Dalstra, P.M. Cattaneo, B. Melsen, Load transfer of miniscrews for orthodontic anchorage, Orthodontics 1 (2004) 53-62.

[17] C.J. Drago, Rates of osseointegration of dental implants with regard to anatomic locations, J. Prosthodont. 1 (1992) 29-31.

[18] S.E. Eckert, P.C. Wollan, Retrospective review of 1170 endosseous implants placed in partially edentulous jaws, J. Prosthet. Dent. 79 (1998) $415-421$.

[19] D.M. Gardner, Platform switching as a means to achieving implant aesthetics, N.Y. State Dent. J. 71 (2005) 34-37.

[20] I.P. Geng, K.B. Tan, G.R. Liu, Application of finite element analysis in implant dentistry: a review of the literature, J. Prosthet. Dent. 85 (2001) $585-598$.

[21] F. Hermann, H. Lerner, A. Palti, Factors influencing the preservation of the periimplant marginal bone, Implant Dent. 16 (2007) 165-175.

[22] J.S. Hermann, J.D. Schoolfield, R.K. Schenk, D. Buser, D.L. Cochran, Influence of the size of the microgap on crestal bone changes around titanium implants. A histometric evaluation of unloaded non-submerged implants in the canine mandible, J. Periodontol. 72 (2001) $1372-1383$.

[23] L. Himmlová, T. Dostálová, A. Kácovský, S. Konvicková, Influence of implant length and diameter on stress distribution: a finite element analysis, J. Prosthet. Dent. 91 (2004) 20-25.

[24] E.P. Holmgren, R.J. Seckinger, L.M. Kilgren, F. Mante, Evaluating parameters of osseointegrated dental implants using finite element analysis - a two dimensional comparative study examining the effects of implant diameter, implant shape, and load direction, J. Oral. Implantol. 24 (1998) 80-88.

[25] H.L. Huang, J.S. Huang, C.C. Ko, J.T. Hsu, C.H. Chang, M.Y.C. Chen, Effect of splinted prosthesis supported a wide implant or two implants: a threedimensional finite element analysis, Clin. Oral Implants Res. 16 (2005) 466-472.

[26] A. Ibrahim, S. Atilla, E. Blent, Influence of occlusal forces on stress distribution in preloaded dental implant screws, J. Prosthet. Dent. 91 (2004) $319-325$.

[27] J.T. Irving, Factors concerning bone loss associated with periodontal disease, J. Dent. Res. 49 (1970) 262-267.

[28] T. Jemt, J. Chai, J. Harnett, M.R. Heath, J.E. Hutton, R.B. Johns, et al, A 5-year prospective multicenter follow-up report on overdentures supported by osseointegrated implants, Int. J. Oral Maxillofac. Implants 11 (1996) 291-298.

[29] S. Joshi, A. Mukherjee, M. Kheur, A. Metha, Mechanical performance of endodontically treated teeth, Finite Elem. Anal. Des. 37 (2001) 587-601.

[30] T. Kitagawa, Y. Tanimoto, K. Nemoto, M. Aida, Influence of cortical bone quality on stress distribution in bone around dental implant, Dent. Mater. J. 24 (2005) 219-224.

[31] H.M. Kyung, H.S. Park, S.M. Bae, J.H. Sung, I.B. Kim, Development of orthodontic micro-implants for intraoral anchorage, J. Clin. Orthod. 37 (2003) $321-$ 328.

[32] R.J. Lazzara, S.S. Porter, Platform switching: a new concept in implant dentistry for controlling postrestorative crestal bone levels, Int. J. Periodontics Restorative Dent. 26 (2006) 9-17.

[33] U. Lekholm, J. Gunne, P. Henry, K. Higuchi, U. Linden, C. Bergstrom, et al, Survival of the Branemark implant in partially edentulous jaws: a 10-year prospective multicenter study, Int. J. Oral Maxillofac. Implants 14 (1999) 639-645.

[34] U. Lekholm, G.A. Zarb, Patient selection and preparation, in: P.I. Branemark, G.A. Zarb, T. Albrektsson (Eds.), Tissue-Integrated Prostheses: Osseointegration in Clinical Dentistry, Quintessence, Chicago, 1985, pp. 199-209.

[35] J.E. Lemon, F. Dietsh-Misch, Biomaterials for dental implants, in: C.E. Misch (Ed.), Contemporary Implant Dentistry, second ed., Mosby, St. Louis, 1999, pp. 271-302.

[36] F. Maceri, M. Martignoni, G. Vairo, Mechanical behaviour of endodontic restorations with multiple prefabricated posts: a finite element approach, J. Biomech. 40 (2007) 2386-2398.

[37] Y. Maeda, J. Miura, I. Taki, M. Sogo, Biomechanical analysis on platform switching: is there any biomechanical rationale?, Clin Oral Implants Res. 18 (2007) 581-584.

[38] J. Mah, F. Bergstrand, Temporary anchorage devices: a status report, J. Clin. Orthod. 39 (2005) 132-136.

[39] R.B. Martin, D.B. Burr, N.A. Sharkey, Skeletal Tissue Mechanics, Springer, New York, 1998.

[40] C.E. Misch, M.W. Bidez, A scientific rationale for dental implant design, in: C.E. Misch (Ed.), Contemporary Implant Dentistry, second ed., Mosby, St. Louis, 1999, pp. 329-343. 
[41] A.N. Natali, R.T. Hart, P.G. Pavan, I. Knets, Mechanics of bone tissue, in: A.N. Natali (Ed.), Dental Biomechanics, Taylor \& Francis, London, 2003 , pp. 1-19.

[42] A.N. Natali, P.G. Pavan, Numerical approach to dental biomechanics, in: A.N. Natali (Ed.), Dental Biomechanics, Taylor \& Francis, London, 2003, pp. 211-239.

[43] A.N. Natali, P.G. Pavan, A comparative analysis based on different strength criteria for evaluation of risk factor for dental implants, Comput. Methods Biomech. Biomed. Eng. 5 (2002) 511-523.

[44] A.N. Natali, P.G. Pavan, A.L. Ruggero, Evaluation of stress induced in peri-implant bone tissue by misfit in multi-implant prosthesis, Dent. Mater. 22 (2006) 388-395.

[45] M.A. Papadopoulos, F. Tarawneh, The use of miniscrew implants for temporary skeletal anchorage in orthodontics: a comprehensive review, Oral Surg. Oral Med. Oral Pathol. Oral Radiol. Endod. 103 (2007) e6-e15.

[46] C.S. Petrie, J.L. Williams, Comparative evaluation of implant designs: influence of diameter, length, and taper on strains in the alveolar crest. A threedimensional finite-element analysis, Clin. Oral Implants Res. 16 (2005) 486-494.

[47] A. Piattelli, A. Scarano, M. Piattelli, Microscopical aspects of failure in osseointegrated dental implants: a report of five cases, Biomaterials 17 (1996) $1235-1241$.

[48] J.Y. Rho, R.B. Ashman, C.H. Turner, Young's modulus of trabecular and cortical bone material: ultrasonic and microtensile measurements, J. Biomech. 26 (1993) 111-119.

[49] A.M. Roos-Jansåker, C. Lindahl, H. Renvert, S. Renvert, Nine- to fourteen-year follow-up of implant treatment. Part I: implant loss and associations to various factors, J. Clin. Periodontol. 33 (2006) 283-289.

[50] X.E. Saab, J.A. Griggs, J.M. Powers, R.L. Engelmeier, Effect of abutment angulation on the strain on the bone around an implant in the anterior maxilla: a finite element study, J. Prosthet. Dent. 97 (2007) 85-92.

[51] Y. Sawako, W. Noriyuki, S. Makoto, O. Takashi, The influence of implant location and length on stress distribution for three-unit implant-supported posterior cantilever fixed partial dentures, J. Prosthet. Dent. 91 (2004) 234-240.

[52] Y.K. Shin, C.H. Han, S.J. Heo, S. Kim, H.J. Chun, Radiographic evaluation of marginal bone level around implants with different neck designs after 1 year, Int. J. Oral Maxillofac. Implants 21 (2006) 789-794.

[53] M.S. Tonetti, Determination of the success and failure of root-form osseointegrated dental implants, Adv. Dent. Res. 13 (1999) $173-180$.

[54] C. Truesdell, R.A. Toupin, The classical field theories, in: S. Flügge (Ed.), Handbuch der physik, vol. III/1, Springer, Berlin, 1960.

[55] G. Vairo, Modellazione geometrica di interventi odontoiatrici per la simulazione del comportamento meccanico di denti curati (in Italian), in: Proc. ISCS National Meeting 2003, Cuen, Napoli, 2005, pp. 177-186.

[56] R.C. Van Staden, H. Guan, Y.C. Loo, Application of the finite element method in dental implant research, Comput. Methods Biomech. Biomed. Eng. 9 (2006) 257-270.

[57] R.J. Weyant, Short-term clinical success of root-form titanium implant systems, J. Evid. Based Dent. Pract. 3 (2003) $127-130$.

[58] O.C. Zienkiewicz, R.L. Taylor, The Finite Element Method, fourth ed., McGraw-Hill, New York, 1998.

[59] O.C. Zienkiewicz, J.Z. Zhu, A simple error estimator and adaptive procedure for practical engineering analysis, Int. J. Numer. Method Eng. 24 (1987) $337-357$. 